ITP-SB-95-08

\title{
Heavy-quark correlations in deep-inelastic electroproduction
}

\author{
B. W. Harris and J. Smith \\ Institute for Theoretical Physics, \\ State University of New York at Stony Brook, \\ Stony Brook, New York 11794-3840, USA
}

March 1995

Submitted to Nuclear Physics B

\begin{abstract}
We have completed the next-to-leading order perturbative QCD corrections to the virtual-photon exclusive differential cross sections for heavy quark production in deep-inelastic electron-proton scattering, i.e. $e+P \rightarrow Q+\bar{Q}+X$. Using these results, we have computed distributions which are sensitive to correlations among the heavy quark, the heavy antiquark, and the associated jet. Some predictions for charm and bottom heavy quark production at the electronproton collider HERA are presented.
\end{abstract}




\section{Introduction}

Order $\alpha_{s}$ QCD corrections to structure functions containing heavy quarks and to single heavy quark inclusive distributions in deep-inelastic electroproduction ( $i . e$. , $\gamma^{*}(q)+P(p) \rightarrow Q\left(p_{1}\right)+X$ where $X$ stands for any final hadronic state allowed by quantum-number conservation and $P(p)$ is a proton of momentum $p$ ) were recently published in [1] and [2], respectively. By combining the next-to-leading order (NLO) heavy quark structure functions with the corresponding light-quark structure functions [3], the heavy quark content of the nucleon has been studied as a function of $Q^{2}=-q^{2}$ and $x=Q^{2} / 2 p \cdot q$ [4]. Event rates for charm production integrated over bins in $x$ and $Q^{2}$ relevant to HERA data have been calculated in [5].

To further the study of deep-inelastic electroproduction of heavy quarks we have recalculated the virtual-photon-parton cross sections of [1] in an exclusive fashion. This enables us to study the single and double differential distributions and correlations among all outgoing particles in the virtual-photon induced reaction $\gamma^{*}+P \rightarrow Q+\bar{Q}+X$ with $X=0$ or 1 jet and to easily incorporate experimental cuts. A short letter, containing a study of invariant mass distributions of heavyquark-heavy-antiquark pairs, has been published [6]. In this paper we present all the calculational details and additional distributions. The transverse and longitudinal photon components are treated separately and the latest CTEQ3 parton densities [7], consistent with the newly released HERA data [8], are used in the kinematic regime appropriate for production of charm and bottom quarks at HERA. We make our predictions at fixed values of $Q^{2}\left(\geq 8.5(\mathrm{GeV} / \mathrm{c})^{2}\right)$ and $x\left(\geq 4.2 \times 10^{-4}\right)$.

Our treatment of electroproduction at HERA extends the existing studies of inclusive QCD corrections in the virtual-photon channel [1], inclusive QCD corrections in the real-photon channel [9], and exclusive QCD corrections in the real-photon channel [10], allowing for an extensive comparison with present and future experimental data. Heavy quark electroproduction has already played an important role in the determination of the gluon distribution function in the proton at low $x$. In fact, a study of the photoproduction and the Weizsäker-Williams electroproduction of charmed mesons has just appeared [11]. Production cross sections of charm and bottom quarks are also relevant in the proposed study of the Cabibbo-KobayashiMaskawa (CKM) matrix elements through the rare decays of $D$ - and $B$ - mesons and the $D \bar{D}$ and $B \bar{B}$ mixing [12]. Our study of deep-inelastic electroproduction is motivated by our desire to avoid complications associated with the parton densities of the photon which must be incorporated when $Q^{2}$ is small. Therefore our results should give a cleaner test of NLO perturbative QCD. By examining distributions and correlations that are trivial at lowest order (for example, the azimuthal angle between the heavy quark and the heavy antiquark) one directly tests the hard scattering cross section that is predicted by perturbative QCD and factorization. Here we should note that this is a fixed order perturbative calculation and suffers from the same problems as all NLO calculations. Hence there are regions in phase space where it will break down. For example, in the above mentioned azimuthal angle distributions, if one looks at the prediction too near the back-to-back configuration there will be an extra enhancement of the cross section due to multiple soft gluon 
emission which is not included in our NLO result.

We stress that here we only consider extrinsic heavy quark production, involving Bethe-Heitler and Compton production from massless partons. For a discussion of intrinsic production, where the heavy quark is considered to be part of the proton's wavefunction, see Brodsky et al. [13]. A variable flavor scheme which joins the extrinsic heavy flavor production picture at $\mu_{\text {phys }} \ll m$ with a light mass parton density picture at $\mu_{\text {phys }} \gg m$ has been discussed by Aivazis et al. [14]. By comparing the fixed flavor scheme calculation of [1] with the variable flavor scheme of [14] it is concluded in 15] that the former yields the most stable and reliable results for $F_{2}\left(x, Q^{2}, m^{2}\right)$ in the threshold region ( $i . e ., Q^{2} \leq 10 m^{2}$ where $m$ is the mass of the heavy quark).

In our exclusive computation we use the subtraction method which is based on the replacement of divergent (collinear or soft) terms in the squared matrix elements by generalized plus distributions. This allows us to isolate the soft and collinear poles within the framework of dimensional regularization without calculating all the phase space integrals in a spacetime dimension $n \neq 4$ as usually required in a traditional single particle inclusive computation. The method has been used in the context of electron-positron annihilation [16], hadroproduction of $Z^{0}$ boson pairs [17], hadroproduction of jets [18], hadroproduction of heavy quarks [19], photoproduction of heavy quarks [10], and hadroproduction of a $W$ boson plus a photon [20]. The essence of the method is nicely described and compared to the also popular phase-space slicing method [21] in the introduction of the paper by Kunszt and Soper [18]. The paper of Mangano et al. [19] contains many useful details. In this method the expressions for the squared matrix elements in the collinear limit appear in a factorized form, where poles in $n-4$ multiply splitting functions and lower order squared matrix elements. The cancellation of collinear singularities is then performed using the factorization theorem [22]. The expressions for the squared matrix elements in the soft limit appear in a factorized form where poles in $n-4$ multiply lower order squared matrix elements. The cancellation of soft singularities takes place upon adding the contributions from the renormalized virtual diagrams. Since the final result is in four-dimensional space time, we can compute all relevant phase space integrations using standard Monte Carlo integration techniques [23] and produce histograms for exclusive, semi-inclusive, or inclusive quantities related to any of the outgoing particles. We can also incorporate any reasonable set of experimental cuts. Our computer code has no small phase space slice parameters and the parameters defining the generalized plus distributions may be tuned to give fast numerical convergence, which is an advantage of using this subtraction method.

We therefore have a new, and more general, calculation of the scale independent (Wilson) coefficient functions (or scale independent partonic cross sections) $c_{k, i}^{(l)}(\eta, \xi), \bar{c}_{k, i}^{(1)}(\eta, \xi)$, and $d_{k, i}^{(1)}(\eta, \xi)$ defined in [1], as functions of $\eta=s / 4 m^{2}-1$, where $s$ is the square of the c. m. energy in the virtual-photon-parton system, and $\xi=Q^{2} / \mathrm{m}^{2}$. We checked the $\eta$ and $\xi$ dependence of these scale independent coefficient functions and the $x$ and $Q^{2}$ dependence of the hadronic structure functions $F_{2}\left(x, Q^{2}, m^{2}\right)$ and $F_{L}\left(x, Q^{2}, m^{2}\right)$, obtained after convolution of the coefficient functions with the light quark and gluon densities in the proton, against the results in 
[1]. We comment on this comparison below. The results for the single quark inclusive transverse momentum and rapidity distributions, $d F_{k}\left(x, Q^{2}, m^{2}, p_{t}\right) / d p_{t}$ and $d F_{k}\left(x, Q^{2}, m^{2}, y\right) / d y(k=G, L)$ respectively, were also checked against the results in [2] to make sure that all our computer programs yield consistent results.

In sect. 2 we introduce our notation used in the calculation of the cross sections for the off-shell photoproduction of heavy quarks. We closely follow the notations of [1] and [10]. In sect. 3 we discuss the analytical results needed for the calculation of the numerical results. The latter are presented in sect. 4. We concentrate on those plots which are dependent on the emission of an extra parton (or jet) and only show single differential distributions. Our conclusions are presented in sect. 5. Kinematics and other useful results are relegated to appendix A. Appendix B contains a discussion of the soft limit of the matrix elements. Appendix $\mathrm{C}$ contains a discussion of the collinear limit of the matrix elements. We have included these appendices to help with the identification of the various pieces of our computer code.

\section{Notation and Born cross section}

In this section we introduce our notation and define the quantities which we will calculate. We then review the Born level results. The kinematics and other useful results are given in appendix A. Following [1], we denote the parton subprocesses contributing to heavy quark production in deep-inelastic electron-hadron scattering by (see fig. 1)

$$
\gamma^{*}(q)+a_{1}\left(k_{1}\right) \rightarrow Q\left(p_{1}\right)+\bar{Q}\left(p_{2}\right)+a_{2}\left(k_{2}\right)+\cdots+a_{j}\left(k_{j}\right),
$$

where the photon momentum is space-like $\left(q^{2}=-Q^{2}<0\right), a_{i}\left(k_{i}\right)$ stand for massless partons $\left(k_{i}^{2}=0\right)$, and $Q(\bar{Q})$ is the heavy (anti) quark $\left(p_{1}^{2}=p_{2}^{2}=m^{2}\right)$. We consider the partonic structure tensor

$$
W_{\mu \nu}=\frac{1}{2 s^{\prime}} \frac{1}{2} \frac{1}{1+\delta_{g a_{1}} \epsilon / 2} K_{a_{1} \gamma}\left[\sum M_{\mu}(2) M_{\nu}^{*}(2) d \Gamma_{2}+\sum M_{\mu}(3) M_{\nu}^{*}(3) d \Gamma_{3}+\cdots\right]
$$

where each term in the square brackets corresponds to a perturbative expansion in $\alpha_{s}$. The terms in the structure tensor are the flux factor $1 / 2 s^{\prime}$, the initial degree of freedom average $1 / 2\left(1+\delta_{g a_{1}} \epsilon / 2\right)$ where $\delta_{g a_{1}}$ is the Kronecker delta function, the color average $K_{a_{1} \gamma}$, the 2 to $j$ body amplitude $M(j)$, the $j$ body phase space $d \Gamma_{j}$, and the sum over initial and final degrees of freedom $\Sigma$. The color average factor has the specific values $K_{g \gamma}=1 /\left(N^{2}-1\right)$ and $K_{q \gamma}=K_{\bar{q} \gamma}=1 / N$, where the number of colors is $N=3$ for $S U(3)$. We work in the context of dimensional regularization with space time dimension $n=4+\epsilon$. Since we integrate over the azimuthal angle between the plane containing the incoming and outgoing leptons and the plane defined by the incoming parton and outgoing heavy quark the partonic tensor (2.2) only has two terms which can be written as

$$
W_{\mu \nu}=d \sigma_{T}\left(-g_{\mu \nu}+\frac{q_{\mu} q_{\nu}}{q^{2}}\right)
$$




$$
+\left(k_{1 \mu}-\frac{k_{1} \cdot q}{q^{2}} q_{\mu}\right)\left(k_{1 \nu}-\frac{k_{1} \cdot q}{q^{2}} q_{\nu}\right)\left(\frac{-4 q^{2}}{s^{\prime 2}}\right)\left(d \sigma_{T}+d \sigma_{L}\right) .
$$

Using the projection operators $g^{\mu \nu}$ and $k_{1}^{\mu} k_{1}^{\nu}$ the partonic cross sections can be written as follows:

$$
\begin{aligned}
d \sigma_{G} & =-\frac{1}{2} \frac{1}{1+\epsilon / 2} g^{\mu \nu} W_{\mu \nu}, \\
d \sigma_{L} & =-\frac{4 q^{2}}{\left(s^{\prime}\right)^{2}} k_{1}^{\mu} k_{1}^{\nu} W_{\mu \nu} .
\end{aligned}
$$

We work with $d \sigma_{G}$ and $d \sigma_{L}$ for convenience and later use these to get the transverse partonic cross section $d \sigma_{T}$ via the relation

$$
d \sigma_{T}=d \sigma_{G}+\frac{1}{2} \frac{1}{1+\epsilon / 2} d \sigma_{L}
$$

For each of the three partonic channels $a_{1}=g, q, \bar{q}$ we expand the corresponding parton cross sections in terms of the number of outgoing particles in reaction (2.1):

$$
d \sigma_{i, a_{1}}=d \sigma_{i, a_{1}}^{(2)}+d \sigma_{i, a_{1}}^{(3)}+\cdots
$$

where $i=G, L$ and

$$
d \sigma_{i, a_{1}}^{(j)}=C_{i, a_{1}} M_{i}^{a_{1}}(j) d \Gamma_{j}
$$

Using eq. (2.2) we find the partonic cross sections are related to the squared matrix elements by

$$
\begin{aligned}
& M_{G}^{a_{1}}(j)=-g^{\mu \nu} \sum M_{\mu}\left(\gamma^{*} a_{1} \rightarrow j\right) M_{\nu}^{*}\left(\gamma^{*} a_{1} \rightarrow j\right), \\
& M_{L}^{a_{1}}(j)=-\frac{4 q^{2}}{\left(s^{\prime}\right)^{2}} k_{1}^{\mu} k_{1}^{\nu} \sum M_{\mu}\left(\gamma^{*} a_{1} \rightarrow j\right) M_{\nu}^{*}\left(\gamma^{*} a_{1} \rightarrow j\right),
\end{aligned}
$$

with

$$
C_{i, a_{1}}=\left(\frac{1}{2} \frac{1}{1+\epsilon / 2}\right) a_{i}(\epsilon)\left(\frac{1}{2 s^{\prime}} \frac{1}{2} \frac{1}{1+\delta_{g a_{1}} \epsilon / 2} K_{a_{1} \gamma}\right),
$$

where $a_{G}(\epsilon)=1$ and $a_{L}(\epsilon)=2(1+\epsilon / 2)$. Note that

$$
C_{i, q}=2 C_{F}(1+\epsilon / 2) C_{i, g}
$$

with the color factor $C_{F}=\left(N^{2}-1\right) / 2 N=4 / 3$ for $S U(3)$. The expressions for $M_{G}^{a_{1}}(j)$ and $M_{L}^{a_{1}}(j)$ were evaluated using the symbolic algebra program FORM [24].

We begin our analysis by considering the lowest order process contributing to reaction (2.1) which is shown in fig. 2. Namely,

$$
\gamma^{*}(q)+g\left(k_{1}\right) \rightarrow Q\left(p_{1}\right)+\bar{Q}\left(p_{2}\right) .
$$

The projections of the matrix element for this reaction are calculated according to eq. (2.9). We calculate in $n=4+\epsilon$ space-time dimensions. Both the $O(\epsilon)$ and $O\left(\epsilon^{2}\right)$ 
terms are needed for a correct treatment of renormalization and mass factorization. Summing over initial and final spins

$$
M_{k}^{g}(2)=8 g^{2} e^{2} e_{H}^{2} N C_{F} B_{k, Q E D},
$$

with $k=G, L$ and

$$
\begin{aligned}
B_{G, Q E D} & =\frac{u_{1}}{t_{1}}+\frac{t_{1}}{u_{1}}+\frac{2 q^{2} s}{t_{1} u_{1}}+\frac{4 m^{2} s^{\prime}}{t_{1} u_{1}}\left(1-\frac{m^{2} s^{\prime}}{t_{1} u_{1}}\right)+\frac{2 m^{2} q^{2}}{t_{1} u_{1}}\left(2-\frac{\left(s^{\prime}\right)^{2}}{t_{1} u_{1}}\right) \\
& +\epsilon\left\{-1+\frac{\left(s^{\prime}\right)^{2}}{t_{1} u_{1}}+\frac{s^{\prime} q^{2}}{t_{1} u_{1}}+\frac{q^{4}}{t_{1} u_{1}}-\frac{m^{2} q^{2}\left(s^{\prime}\right)^{2}}{t_{1}^{2} u_{1}^{2}}\right\}+\epsilon^{2} \frac{\left(s^{\prime}\right)^{2}}{4 t_{1} u_{1}} \\
B_{L, Q E D} & =\frac{-4 q^{2}}{\left(s^{\prime}\right)^{2}}\left[s-\frac{m^{2}\left(s^{\prime}\right)^{2}}{t_{1} u_{1}}\right] .
\end{aligned}
$$

The kinematic variables are defined in appendix A. Here $g$ and $e$ are the strong and electromagnetic coupling constants respectively and $e_{H}$ is the charge of the heavy quark in units of $e$. The lowest order cross section now follows directly from eq. (2.8).

\section{Order $\alpha_{s}$ corrections}

In this section we describe the calculation of the NLO corrections to the lowest order gluon fusion cross sections which were derived in the last section. As mentioned in the introduction, we proceed via the subtraction method wherein one extracts the soft and/or collinear singularities without integration over final state particles $Q$ and $a_{2}$ as done in the previous single particle inclusive calculation [1], [2]. This allows us to plot correlations between final state particles which will be presented in sec. 4. We first discuss the gluon-bremsstrahlung reaction in detail and then, in the following subsection, the light quark contributions. The kinematics of the three

body final states are discussed in appendix A, which also contains the definitions of the variables used here.

\section{$3.1 \gamma^{*} g$ Channel}

The gluon-bremsstrahlung reaction, shown in fig. 3 , is

$$
\gamma^{*}(q)+g\left(k_{1}\right) \rightarrow g\left(k_{2}\right)+Q\left(p_{1}\right)+\bar{Q}\left(p_{2}\right) .
$$

We begin by writing the partonic cross sections as

$$
\begin{aligned}
d \sigma_{i, g}^{(3)} & =C_{i, g} M_{i}^{g}(3) d \Gamma_{3} \\
& =C_{i, g} f_{i}^{g}\left(x, y, \theta_{1}, \theta_{2}\right) \overline{d \Gamma_{3}}
\end{aligned}
$$

where

$$
f_{i}^{g}\left(x, y, \theta_{1}, \theta_{2}\right) \equiv t^{\prime}\left(u^{\prime}-q^{2} s_{5} / s\right) M_{i}^{g}(3)
$$




$$
\overline{d \Gamma_{3}} \equiv \frac{d \Gamma_{3}}{t^{\prime}\left(u^{\prime}-q^{2} s_{5} / s\right)} .
$$

We suppress all arguments of $f_{i}^{g}$ other than $x, y, \theta_{1}, \theta_{2}$ for notational convenience. These are the variables we integrate over to calculate the partonic cross sections for reaction (3.1). The subtraction method proceeds by multiplication of the squared matrix element, and division of the phase space, by invariants (or combinations thereof) that vanish in the soft and/or collinear limit. The invariants are chosen to cancel the singularities in the squared matrix elements due to the propagator(s), thus giving finite functions $f_{i}^{g}\left(x, y, \theta_{1}, \theta_{2}\right)$ which can safely be implemented in a computer program. The modified phase space $\overline{d \Gamma}_{3}$ is expanded using generalized plus distributions allowing extraction of soft and/or collinear poles in $n-4$ which are cancelled as usual.

In this particular case, as the photon is virtual, and the heavy quarks are massive, the only collinear singularity comes from the collinear emission of a gluon from the incoming gluon as shown in fig. $3(\mathrm{~d})$. The squared matrix element $M_{i}^{g}(3)$ has a $1 /(1+y)$ singularity when $y \rightarrow-1$, therefore it suffices to multiply by $t^{\prime} \propto(1+y)(1-x)$ to get a result that is finite as $y \rightarrow-1$. Similarly, the emission of a soft gluon corresponds to a $1 /(1-x)^{2}$ singularity in $M_{i}^{g}(3)$ as $x \rightarrow 1$ so we choose to multiply by an additional factor of $\left(u^{\prime}-q^{2} s_{5} / s\right) \propto(1-x)$ to get a result that is finite as $x \rightarrow 1$.

We now proceed to show the cancellation of the singularities and derive the renormalized partonic cross sections. Substituting the expressions for $t^{\prime}$ and $u^{\prime}$ found in appendix A and using eq. (A.12) we find

$$
\overline{d \Gamma_{3}}=\frac{2}{\pi} H N d \Gamma_{2}^{(5)}\left(s^{\prime}\right)^{-1+\epsilon / 2}\left(\frac{s^{\prime}}{s}\right)^{-1+\epsilon / 2}(1-x)^{-1+\epsilon}\left(1-y^{2}\right)^{-1+\epsilon / 2} d y \sin ^{\epsilon} \theta_{2} d \theta_{2},
$$

where all quantities are defined in appendix A. We now begin to extract the poles, by expanding the $(1-x)^{-1+\epsilon}$ piece in terms of the generalized plus distributions defined in appendix A. Using eq. (A.18) one finds

$$
\begin{aligned}
d \sigma_{i, g}^{(3)} & =\frac{2}{\pi} C_{i, g} H N d \Gamma_{2}^{(5)}\left(s^{\prime}\right)^{-1+\epsilon / 2}\left(\frac{s^{\prime}}{s}\right)^{-1+\epsilon / 2} \delta(1-x)\left[\frac{1}{\epsilon}+2 \ln \tilde{\beta}+2 \epsilon \ln ^{2} \tilde{\beta}\right] \\
& \times\left(1-y^{2}\right)^{-1+\epsilon / 2} d y \sin ^{\epsilon} \theta_{2} d \theta_{2} f_{i}^{g}\left(x, y, \theta_{1}, \theta_{2}\right) \\
& +\frac{2}{\pi} C_{i, g} H N d \Gamma_{2}^{(5)}\left(s^{\prime}\right)^{-1+\epsilon / 2}\left(\frac{s^{\prime}}{s}\right)^{-1+\epsilon / 2}\left[\left(\frac{1}{1-x}\right)_{\tilde{\rho}}+\epsilon\left(\frac{\ln (1-x)}{1-x}\right)_{\tilde{\rho}}\right] \\
& \times\left(1-y^{2}\right)^{-1+\epsilon / 2} d y \sin ^{\epsilon} \theta_{2} d \theta_{2} f_{i}^{g}\left(x, y, \theta_{1}, \theta_{2}\right) .
\end{aligned}
$$

The $\delta(1-x) / \epsilon$ piece in the first term is a soft singularity now regulated dimensionally. In the first piece we use $d \Gamma_{2}^{(5)} \delta(1-x)=\delta(1-x) d \Gamma_{2} d x$ while in the second piece we expand $\left(1-y^{2}\right)^{-1+\epsilon / 2}$ using eq. (A.17) to find,

$$
d \sigma_{i, g}^{(3)}=d \sigma_{i, g}^{(s)}+d \sigma_{i, g}^{(c-)}+d \sigma_{i, g}^{(f)}
$$

where

$$
d \sigma_{i, g}^{(s)}=\frac{2}{\pi} C_{i, g} H N d \Gamma_{2}\left(s^{\prime}\right)^{-1+\epsilon / 2}\left(\frac{s^{\prime}}{s}\right)^{-1+\epsilon / 2}\left[\frac{1}{\epsilon}+2 \ln \tilde{\beta}+2 \epsilon \ln ^{2} \tilde{\beta}\right] f_{i}^{g}\left(\theta_{1}\right),
$$


with

$$
f_{i}^{g}\left(\theta_{1}\right)=\int \delta(1-x) d x\left(1-y^{2}\right)^{-1+\epsilon / 2} d y \sin ^{\epsilon} \theta_{2} d \theta_{2} f_{i}^{g}\left(x, y, \theta_{1}, \theta_{2}\right)
$$

and

$$
\begin{aligned}
d \sigma_{i, g}^{(c-)} & =\frac{2}{\pi} C_{i, g} H N d \Gamma_{2}^{(5)}\left(s^{\prime}\right)^{-1+\epsilon / 2}\left(\frac{s^{\prime}}{s}\right)^{-1+\epsilon / 2}\left[\left(\frac{1}{1-x}\right)_{\tilde{\rho}}+\epsilon\left(\frac{\ln (1-x)}{1-x}\right)_{\tilde{\rho}}\right] \\
& \times \delta(1+y)\left(\frac{1}{\epsilon}+\frac{1}{2} \ln 2 \omega\right) d y \sin ^{\epsilon} \theta_{2} d \theta_{2} f_{i}^{g}\left(x, y, \theta_{1}, \theta_{2}\right)
\end{aligned}
$$

and

$$
\begin{aligned}
d \sigma_{i, g}^{(f)} & =\frac{1}{\pi} C_{i, g} H N d \Gamma_{2}^{(5)}\left(s^{\prime}\right)^{-1+\epsilon / 2}\left(\frac{s^{\prime}}{s}\right)^{-1+\epsilon / 2}\left[\left(\frac{1}{1-x}\right)_{\tilde{\rho}}+\epsilon\left(\frac{\ln (1-x)}{1-x}\right)_{\tilde{\rho}}\right] \\
& \times\left[\left(\frac{1}{1+y}\right)_{\omega}+\left(\frac{1}{1-y}\right)_{\omega}\right] d y \sin ^{\epsilon} \theta_{2} d \theta_{2} f_{i}^{g}\left(x, y, \theta_{1}, \theta_{2}\right) .
\end{aligned}
$$

According to the factorization theorem [22] the cross section for scattering of a virtual-photon off a hadron $H$ of momentum $p$ is

$$
d \sigma_{H}(p)=\sum_{i} \int_{0}^{1} d \hat{\sigma}_{i}(\xi p) \phi_{i / H}\left(\xi, \mu_{f}^{2}\right) d \xi,
$$

where the sum runs over all partons in $H$ and we have suppressed the polarization index temporarily. Here $\phi_{i / H}\left(\xi, \mu_{f}^{2}\right)$ is the probability density for finding parton $i$ in hadron $H$ with momentum fraction between $\xi$ and $\xi+d \xi$ at scale $\mu_{f}$. Noting that the infrared safe $d \hat{\sigma}_{i}$ is independent of the external hadron, we are free to set $H=j$, where $j$ represents a parton. Then the partonic cross sections satisfy

$$
d \sigma_{j}\left(k_{1}\right)=\sum_{i} \int_{0}^{1} d \hat{\sigma}_{i}\left(x k_{1}\right) \phi_{i / j}\left(x, \mu_{f}^{2}\right) d x .
$$

Up to first order in $\alpha_{s}$

$$
\phi_{i / j}\left(x, \mu_{f}^{2}\right)=\delta_{i j} \delta(1-x)+\frac{\alpha_{s}}{2 \pi}\left[P_{i j}(x) \frac{2}{\epsilon}+f_{i j}\left(x, \mu_{f}^{2}\right)\right],
$$

where $P_{i j}(x)$ denote the Altarelli-Parisi splitting functions 25] and the functions $f_{i j}\left(x, \mu_{f}^{2}\right)$ depend on the mass factorization scheme chosen. In the $\overline{\mathrm{MS}}$ scheme

$$
f_{i j}\left(x, \mu_{f}^{2}\right)=P_{i j}(x)\left(\gamma_{E}-\ln 4 \pi+\ln \mu_{f}^{2} / \mu^{2}\right),
$$

where $\gamma_{E}$ is the Euler-Mascheroni constant. In this paper we choose the mass factorization scale $\mu_{f}$ to be equal to the renormalization scale $\mu$ so that the last term in eq. (3.15) is zero. Expanding $d \sigma_{j}$ and $d \hat{\sigma}_{i}$ in powers of $\alpha_{s}$ gives

$$
d \sigma_{j}\left(k_{1}\right)=d \sigma_{j}^{(0)}\left(k_{1}\right)+d \sigma_{j}^{(1)}\left(k_{1}\right)+\cdots
$$




$$
d \hat{\sigma}_{i}\left(x k_{1}\right)=d \hat{\sigma}_{i}^{(0)}\left(x k_{1}\right)+d \hat{\sigma}_{i}^{(1)}\left(x k_{1}\right)+\cdots .
$$

Substituting eqs. (3.14), (3.15), (3.16), and (3.17) into eq. (3.13) and equating powers of $\alpha_{s}$ we obtain

$$
\begin{aligned}
d \hat{\sigma}_{j}^{(0)}\left(k_{1}\right) & =d \sigma_{j}^{(0)}\left(k_{1}\right), \\
d \hat{\sigma}_{j}^{(1)}\left(k_{1}\right) & =d \sigma_{j}^{(1)}\left(k_{1}\right)-\frac{\alpha_{s}}{2 \pi} \sum_{i} \int_{0}^{1} d x d \sigma_{i}^{(0)}\left(x k_{1}\right) P_{i j}(x) \frac{2}{\bar{\epsilon}},
\end{aligned}
$$

where $2 / \bar{\epsilon}=2 / \epsilon+\gamma_{E}-\ln 4 \pi$.

In the collinear limit $y \rightarrow-1$ we show in appendix B that

$$
f_{i}^{g}\left(x,-1, \theta_{1}, \theta_{2}\right)=f_{i}^{g}\left(x, \theta_{1}\right)+\tilde{f}_{i}^{g}\left(x, \theta_{1}, \theta_{2}\right),
$$

where

$$
\int_{0}^{\pi} \tilde{f}_{i}^{g}\left(x, \theta_{1}, \theta_{2}\right) \sin ^{\epsilon} \theta_{2} d \theta_{2}=0
$$

and

$$
\begin{aligned}
f_{i}^{g}\left(x, \theta_{1}\right) & =512 \pi^{2} \mu^{-2 \epsilon} \alpha_{s}^{2} e^{2} e_{H}^{2} N C_{A} C_{F} \frac{\left(s^{\prime}\right)^{2}}{s} \\
& \times \frac{1-x}{x}\left[x(1-x)+\frac{x}{1-x}+\frac{1-x}{x}\right] B_{i, Q E D}\left(x k_{1}\right) .
\end{aligned}
$$

Performing the $y$ and $\theta_{2}$ integrations in eq. (3.10) using eqs. (3.20), (3.21), and (3.22) we find

$$
\begin{aligned}
d \sigma_{i, g}^{(c-)} & =32 C_{i, g} \mu^{-\epsilon} \alpha_{s}^{2} e^{2} e_{H}^{2} N C_{A} C_{F} d \Gamma_{2}^{(5)} \\
& \times\left\{\frac{2}{\bar{\epsilon}}\left(\frac{1}{1-x}\right)_{\tilde{\rho}}+2\left(\frac{\ln (1-x)}{1-x}\right)_{\tilde{\rho}}+\left(\frac{1}{1-x}\right)_{\tilde{\rho}}\left[\ln \frac{s^{\prime}}{\mu^{2}}+\ln \frac{s^{\prime}}{s}+\ln \frac{\omega}{2}\right]\right\} \\
& \times \frac{1-x}{x}\left[x(1-x)+\frac{x}{1-x}+\frac{1-x}{x}\right] B_{i, Q E D}\left(x k_{1}\right) .
\end{aligned}
$$

Appealing to eq. (3.19) and reinstating the polarization index $i$ we find

$$
\begin{aligned}
d \hat{\sigma}_{i, g}^{(v)}+d \hat{\sigma}_{i, g}^{(s)}+d \hat{\sigma}_{i, g}^{(c-)} & =d \sigma_{i, g}^{(v)}+d \sigma_{i, g}^{(s)}+d \sigma_{i, g}^{(c-)} \\
& -\frac{\alpha_{s}}{2 \pi} \int d x d \sigma_{i, g}^{(0)}\left(x k_{1}\right) P_{g g}(x) \frac{2}{\bar{\epsilon}},
\end{aligned}
$$

where we have used $d \sigma_{i, q}^{(0)}=d \sigma_{i, \bar{q}}^{(0)}=0$. The Born level cross sections $d \sigma_{i, g}^{(0)}$ are given by eqs. (2.8) and 2.13) and the virtual corrections denoted by $d \sigma_{i, g}^{(v)}$ are discussed below. It is well known that 25]

$$
\begin{aligned}
P_{g g}(x) & =2 C_{A}\left[\frac{x}{(1-x)_{+}}+\frac{1-x}{x}+x(1-x)\right]+\left(\frac{11}{6} C_{A}-\frac{2}{3} T_{F} n_{l f}\right) \delta(1-x), \\
& =2 C_{A}\left[\frac{x}{(1-x)_{\tilde{\rho}}}+\frac{1-x}{x}+x(1-x)\right] \\
& +\left(\frac{11}{6} C_{A}-\frac{2}{3} T_{F} n_{l f}+4 C_{A} \ln \tilde{\beta}\right) \delta(1-x),
\end{aligned}
$$


where $T_{F}=1 / 2$, and $n_{l f}$ is the number of light quarks. In the second line we have exchanged the usual plus distribution for the more general $\tilde{\rho}$ distribution and defined $\tilde{\beta}=\sqrt{1-\tilde{\rho}}$. Cancelling the pole in eq. (3.23) by using eq. (3.24) gives the "factorized collinear" term

$$
\begin{aligned}
d \hat{\sigma}_{i, g}^{(c-)}= & 32 C_{i, g} \alpha_{s}^{2} e^{2} e_{H}^{2} N C_{A} C_{F} B_{i, Q E D}\left(x k_{1}\right) d \Gamma_{2}^{(5)}\left[(1-x)^{2}+1+\frac{(1-x)^{2}}{x^{2}}\right] \\
& \times\left[\left(\frac{1}{1-x}\right)_{\tilde{\rho}}\left(\ln \frac{s^{\prime}}{\mu^{2}}+\ln \frac{s^{\prime}}{s}+\ln \frac{\omega}{2}\right)+2\left(\frac{\ln (1-x)}{1-x}\right)_{\tilde{\rho}}\right]
\end{aligned}
$$

In the limit $Q^{2}=0$ this result reduces to formula (2.45) of [10] when one identifies $M_{\gamma g}^{(b)}=32 C_{i, g} \pi \alpha_{s} e^{2} e_{H}^{2} N C_{F} B_{G, Q E D}$. The remaining poles in eq. (3.24) cancel when the soft and virtual cross sections are added according to

$$
\begin{aligned}
d \hat{\sigma}_{i, g}^{(s+v)} & =d \sigma_{i, g}^{(v)}+d \sigma_{i, g}^{(s)} \\
& -16 C_{i, g} \alpha_{s}^{2} \mu^{-\epsilon} e^{2} e_{H}^{2} N C_{F} d \Gamma_{2} \frac{2}{\bar{\epsilon}}\left(\frac{11}{6} C_{A}-\frac{2}{3} T_{F} n_{l f}+4 C_{A} \ln \tilde{\beta}\right) B_{i, Q E D}\left(k_{1}\right) .
\end{aligned}
$$

The soft cross sections $d \sigma_{i, g}^{(s)}$, which follow from eq. (3.8), are given in appendix B. The one loop order $e g^{3}$ diagrams for the virtual corrections to the reaction (2.12) are shown in fig. 4 . The interference of these diagrams with the lowest order diagrams of fig. 2 were calculated in [1] using the renormalization scheme of [26] and are available as FORM code. Using (3.27) we analytically checked the cancellation of the singularities. Also available from [1] as FORTRAN code is the analog of eq. (3.27) using the $\Delta$ prescription [27] for dividing the phase space into soft and collinear regions. The components of (3.27) in the $\Delta$ prescription and the subtraction method differ by finite terms. Therefore, we modified the original FORTRAN code by adding and subtracting appropriate finite pieces. In fact, the soft finite pieces to subtract off are given in eqs. (3.24) and (3.25) of [1], and the pieces to add on are the finite pieces of eqs. $(\overline{B .16})$ and $(\overline{B .17})$ of appendix B. Note that there is a typographical error in eq. (3.25) of [1]; the sign of the $\ln ^{2} r_{s}$ term should be negative not positive.

Finally the three body contributions $d \sigma_{i, g}^{(f)}$ are finite in $n=4$ space-time dimensions and a short calculation from (3.11) shows they may be written as

$$
\begin{aligned}
d \sigma_{i, g}^{f}= & 2\left(\frac{1}{16 \pi^{2}}\right)^{2} C_{i, g} \beta_{5} \frac{s}{\left(s^{\prime}\right)^{2}}\left(\frac{1}{1-x}\right)_{\tilde{\rho}}\left(\frac{1}{1+y}\right)_{\omega} \frac{1}{1-y} \\
& \times f_{i}^{g}\left(x, y, \theta_{1}, \theta_{2}\right) d x d y \sin \theta_{1} d \theta_{1} d \theta_{2}
\end{aligned}
$$

where $\beta_{5}=\sqrt{1-4 m^{2} / s_{5}}$. The finite functions $f_{i}^{g}\left(x, y, \theta_{1}, \theta_{2}\right)$ follow from the definition (3.3), eq. (2.9), and the diagrams in fig. 3. Summarizing, we have the final result

$$
d \hat{\sigma}_{i, g}=d \sigma_{i, g}^{(0)}+d \hat{\sigma}_{i, g}^{(s+v)}+d \hat{\sigma}_{i, g}^{(c-)}+d \sigma_{i, g}^{(f)},
$$

that is finite in $n=4$ space-time dimensions and is to be used in eq. (3.12) to make predictions. Each of the last three terms in (3.29) individually depends on $\tilde{\rho}$ and $\omega$. 
However, the sum is independent of them as it must be because the decomposition (3.29) follows directly from eqs. (A.17) and (A.18). This provides a strong check on our computer code.

\section{$3.2 \gamma^{*} q$ and $\gamma^{*} \bar{q}$ Channels}

Analysis of the partonic reaction(s)

$$
\gamma^{*}(q)+q\left(k_{1}\right)\left(\bar{q}\left(k_{1}\right)\right) \rightarrow q\left(k_{2}\right)\left(\bar{q}\left(k_{2}\right)\right)+Q\left(p_{1}\right)+\bar{Q}\left(p_{2}\right),
$$

proceeds as above only this time there are no soft or virtual contributions making the analysis much simpler. This time we write the partonic cross sections as

$$
\begin{aligned}
d \sigma_{i, q}^{(3)} & =C_{i, q} M_{i}^{q}(3) d \Gamma_{3} \\
& =C_{i, q} f_{i}^{q}\left(x, y, \theta_{1}, \theta_{2}\right) \overline{d \Gamma_{3}}
\end{aligned}
$$

where

$$
f_{i}^{q}\left(x, y, \theta_{1}, \theta_{2}\right) \equiv t^{\prime} M_{i}^{q}(3),
$$

and

$$
\overline{d \Gamma_{3}} \equiv d \Gamma_{3} / t^{\prime} .
$$

Replacing the divergent factor $(1+y)^{-1+\epsilon / 2}$ in $\overline{d \Gamma_{3}}$ by eq. (A.16) one obtains the following decomposition:

$$
d \sigma_{i, q}^{(3)}=d \sigma_{i, q}^{(c-)}+d \sigma_{i, q}^{(f)},
$$

with

$$
\begin{aligned}
d \sigma_{i, q}^{(c-)} & =-\frac{1}{\pi} C_{i, q} H N d \Gamma_{2}^{(5)}\left(s^{\prime}\right)^{\epsilon / 2}\left(\frac{s^{\prime}}{s}\right)^{\epsilon / 2}(1-x)^{\epsilon}(1-y)^{\epsilon / 2} \\
& \times \delta(1+y)\left(\frac{2}{\epsilon}+\ln \omega\right) d y \sin ^{\epsilon} \theta_{2} d \theta_{2} f_{i}^{q}\left(x, y, \theta_{1}, \theta_{2}\right),
\end{aligned}
$$

and

$$
\begin{aligned}
d \sigma_{i, q}^{(f)} & =-\frac{1}{\pi} C_{i, q} H N d \Gamma_{2}^{(5)}\left(s^{\prime}\right)^{\epsilon / 2}\left(\frac{s^{\prime}}{s}\right)^{\epsilon / 2}(1-x)^{\epsilon}(1-y)^{\epsilon / 2} \\
& \times\left(\frac{1}{1+y}\right)_{\omega} d y \sin ^{\epsilon} \theta_{2} d \theta_{2} f_{i}^{q}\left(x, y, \theta_{1}, \theta_{2}\right) .
\end{aligned}
$$

As in the gluon-bremsstrahlung reaction (see appendix $\mathrm{C}$ )

$$
f_{i}^{q}\left(x,-1, \theta_{1}, \theta_{2}\right)=f_{i}^{q}\left(x, \theta_{1}\right)+\tilde{f}_{i}^{q}\left(x, \theta_{1}, \theta_{2}\right),
$$

with

$$
\int_{0}^{\pi} \tilde{f}_{i}^{q}\left(x, \theta_{1}, \theta_{2}\right) \sin ^{\epsilon} \theta_{2} d \theta_{2}=0
$$


In this case we find that

$$
\begin{aligned}
f_{i}^{q}\left(x, \theta_{1}\right) & =-128 \pi^{2} \mu^{-2 \epsilon} \alpha_{s}^{2} e^{2} e_{H}^{2} N C_{F}(1+\epsilon / 2)^{-1} \\
& \times\left[\frac{1+(1-x)^{2}+\epsilon x^{2} / 2}{x^{2}}\right] B_{i, Q E D}\left(x k_{1}\right) .
\end{aligned}
$$

Appealing to eq. (3.19) with 25]

$$
P_{g q}(x)=C_{F}\left[\frac{1+(1-x)^{2}}{x}\right],
$$

one finds

$$
d \hat{\sigma}_{i, q}^{(3)}=d \hat{\sigma}_{i, q}^{(c-)}+d \sigma_{i, q}^{(f)}
$$

with

$$
\begin{aligned}
d \hat{\sigma}_{i, q}^{(c-)} & =8 C_{i, q} \alpha_{s}^{2} e^{2} e_{H}^{2} N C_{F} B_{i, Q E D}\left(x k_{1}\right) d \Gamma_{2}^{(5)} \\
& \times\left\{1+\frac{1+(1-x)^{2}}{x^{2}}\left[\ln \frac{s^{\prime}}{\mu^{2}}+\ln \frac{s^{\prime}}{s}+\ln \frac{\omega}{2}+2 \ln (1-x)\right]\right\} \\
d \sigma_{i, q}^{(f)} & =-\left(\frac{1}{16 \pi^{2}}\right)^{2} C_{i, q} \beta_{5} f_{i}^{q}\left(x, y, \theta_{1}, \theta_{2}\right)\left(\frac{1}{1+y}\right)_{\omega} d x d y \sin \theta_{1} d \theta_{1} d \theta_{2}
\end{aligned}
$$

The finite functions $f_{i}^{q}\left(x, y, \theta_{1}, \theta_{2}\right)$ follow from the definition (3.32), eq. (2.9), and the diagrams in fig. 5. In the limit $Q^{2}=0$, the "factorized collinear" cross sections for the quark channel $d \hat{\sigma}_{i, q}^{(c-)}$ reduces to formula (2.62) of [10] when one identifies $M_{\gamma g}^{(b)}=32 C_{i, g} \pi \alpha_{s} e^{2} e_{H}^{2} N C_{F} B_{G, Q E D}$ and uses eq. (2.11). This completes the analysis of the quark induced reaction. The analysis of the antiquark induced reaction is completely analogous.

As the quark channel only contains collinear singularities, we will use it to illustrate how the generalized plus distributions are implemented numerically and how the $\omega$ dependence disappears in the sum (3.41). To this end consider

$$
\begin{aligned}
d \sigma_{i, q}^{(f)} & \sim \int_{-1}^{1} d y f(y)\left(\frac{1}{1+y}\right)_{\omega} \\
& =\int_{-1}^{-1+\omega} d y f(y)\left(\frac{1}{1+y}\right)_{\omega}+\int_{-1+\omega}^{1} d y f(y)\left(\frac{1}{1+y}\right)_{\omega} \\
& =\int_{-1}^{-1+\omega} d y \frac{f(y)-f(-1)}{1+y}+\int_{-1+\omega}^{1} d y \frac{f(y)}{1+y} \\
& =\int_{-1}^{1} d y \frac{f(y)}{1+y}-\int_{-1}^{-1+\omega} d y \frac{f(-1)}{1+y}
\end{aligned}
$$

where we have suppressed all indices and arguments of $f_{i}^{q}\left(x, y, \theta_{1}, \theta_{2}\right)$ other than $y$. In the bottom line we see that the infinity encountered at the lower integration limit $y=-1$ is cancelled in the sum of the two integrals. In practice one can only 
reasonably take the lower limit to be $-1+\delta$ where $\delta \sim 10^{-7}$ in double precision FORTRAN before round off errors enter. None the less, the final results are stable with respect to the variation of $\delta$ in the range $10^{-5}$ to $10^{-7}$. The upper limit of the second integral gives a contribution $f(-1) \ln \omega$ which cancels against the $\ln \omega$ term in $d \hat{\sigma}_{i, q}^{(c-)}$. The first integral in the bottom line is commonly called the "event" and has a positive definite weight. The second integral plus the factorized collinear contribution is commonly called the "counter-event" and may have either positive or negative weight. The implementation for the gluon channel is similar but more complicated due to the presence of the soft-virtual terms.

\section{Results}

\subsection{Virtual-photon-parton cross sections}

In this section we discuss the virtual-photon-parton cross sections and compare them with the results of [1]. The total cross sections are obtained by integration so that

$$
\hat{\sigma}_{k, i}=\int d \hat{\sigma}_{k, i}
$$

where $d \hat{\sigma}_{k, i}$ are given as eqs. (3.29), and (3.41). Choosing a renormalization scheme where the heavy quarks in the gluon self-energy loops decouple in the limit of small momenta flowing into the loop [26], we express the perturbative expansion of the virtual photon-parton cross section in terms of scaling functions as follows:

$$
\hat{\sigma}_{k, i}\left(s, q^{2}, m^{2}\right)=\frac{\alpha \alpha_{s}}{m^{2}}\left[f_{k, i}^{(0)}(\eta, \xi)+4 \pi \alpha_{s}\left\{f_{k, i}^{(1)}(\eta, \xi)+\bar{f}_{k, i}^{(1)}(\eta, \xi) \ln \frac{\mu_{f}^{2}}{m^{2}}\right\}\right]
$$

where

$$
\eta=\frac{s}{4 m^{2}}-1, \quad \xi=\frac{Q^{2}}{m^{2}}
$$

with $s$ the square of the c. m. energy in the virtual-photon-parton system and $\mu_{f}^{2}$ the mass factorization scale. Since the $f_{k, i}^{(l)}(\eta, \xi)$ depend on the electric charges of the heavy and/or light quarks we extract these charges and define new functions via

$$
\begin{aligned}
f_{k, g}^{(l)}(\eta, \xi) & =e_{H}^{2} c_{k, g}^{(l)}(\eta, \xi), \\
f_{k, q}^{(1)}(\eta, \xi) & =e_{H}^{2} c_{k, q}^{(1)}(\eta, \xi)+e_{H} e_{L} o_{k, q}^{(1)}(\eta, \xi)+e_{L}^{2} d_{k, q}^{(1)}(\eta, \xi),
\end{aligned}
$$

together with the corresponding formulae for the coefficient of the mass factorization term. Note that the $o_{k, q}^{(1)}(\eta, \xi)$ vanish after integration to form the total photonparton cross sections so they were not needed in [1]. However, now they must be retained when plotting distributions. Further, $d_{k, q}^{(1)}(\eta, \xi)$ can only be evaluated at $\xi=0$ provided the additional collinear divergence which arises when the photon goes on-mass-shell is mass factorized. As we only consider $Q^{2} \geq 8.5(\mathrm{GeV} / \mathrm{c})^{2}$ we do not perform this factorization although it was performed in [1] and checked against 
the photoproduction limit in $[9] . m=4.75\left(\mathrm{GeV} / c^{2}\right)$ i.e. the corrected version of fig. 11(b) of

Upon integration of the eqs. (3.29), and (3.41) we find agreement with all the plots shown in [1] except for discrepancies with three figures, namely, fig. 9(b), fig. 11(a), and fig. 11(b). The correct plots are reproduced here as fig. 6, fig. 7(a), and fig. 7(b), respectively. This checking also uncovered minor errors in the previous FORTRAN programs for the inclusive calculations which have now been corrected. Fortunately, they were all in the virtual-photon-quark channels so were not significant numerically and do not alter any of the other plots or results in references [1], [2], and [5].

\subsection{Hadronic structure functions and correlations}

Recalling that the probability density is related to the momentum density via $f_{i / H}\left(\xi, \mu_{f}^{2}\right)=\xi \phi_{i / H}\left(\xi, \mu_{f}^{2}\right)$ we write (3.12) as

$$
d \sigma_{\gamma^{*} H}(p)=\sum_{i} \int_{0}^{1} \frac{d \xi}{\xi} d \hat{\sigma}_{i}(\xi p) f_{i / H}\left(\xi, \mu_{f}^{2}\right) .
$$

We now specialize to the case where $H$ is a proton, as in the case of HERA. Using the relations

$$
F_{k}=\frac{Q^{2}}{4 \pi^{2} \alpha} \sigma_{k}
$$

where $k=2, L$ with $\sigma_{2}=\sigma_{G}+3 \sigma_{L} / 2$, and the relations for the scaling functions (4.2), and (4.4), we find

$$
\begin{aligned}
F_{k}\left(x, Q^{2}, m^{2}\right)= & \frac{Q^{2} \alpha_{s}\left(\mu^{2}\right)}{4 \pi^{2} m^{2}} \int_{\xi_{\min }}^{1} \frac{d \xi}{\xi}\left[e_{H}^{2} f_{g / P}\left(\xi, \mu^{2}\right) c_{k, g}^{(0)}\right] \\
& +\frac{Q^{2} \alpha_{s}^{2}\left(\mu^{2}\right)}{\pi m^{2}} \int_{\xi_{\min }}^{1} \frac{d \xi}{\xi}\left\{e_{H}^{2} f_{g / P}\left(\xi, \mu^{2}\right)\left(c_{k, g}^{(1)}+\bar{c}_{k, g}^{(1)} \ln \frac{\mu^{2}}{m^{2}}\right)\right. \\
& \left.+\sum_{i=q, \bar{q}} f_{i / P}\left(\xi, \mu^{2}\right)\left[e_{H}^{2}\left(c_{k, i}^{(1)}+\bar{c}_{k, i}^{(1)} \ln \frac{\mu^{2}}{m^{2}}\right)+e_{i}^{2} d_{k, i}^{(1)}+e_{i} e_{H} o_{k, i}^{(1)}\right]\right\},
\end{aligned}
$$

where $k=2, L$. We have set $\mu_{f}=\mu$ and shown the $\mu^{2}$ dependence of the running coupling $\alpha_{s}$ explicitly. The lower boundary on the integration is given by $\xi_{\min }=$ $x\left(4 m^{2}+Q^{2}\right) / Q^{2}$. This formula yields the standard heavy quark structure functions $F_{2}\left(x, Q^{2}, m^{2}\right)$ and $F_{L}\left(x, Q^{2}, m^{2}\right)$ for electron proton scattering, and we will present results as differentials of these functions.

From the formalism described in the previous section we are left with events of positive weight and counter-events of either positive or negative weight. Our program outputs the final state four vectors of the event and counter-event together with the corresponding weight. We histogram these into bins to produce differential distributions. 
We note that the inclusive distributions in the heavy quark transverse momentum, (i.e., $d F_{2}\left(x, Q^{2}, m^{2}, p_{t}\right) / d p_{t}$ and $\left.d F_{L}\left(x, Q^{2}, m^{2}, p_{t}\right) / d p_{t}\right)$ and in the heavy quark rapidity ( i.e., $d F_{2}\left(x, Q^{2}, m^{2}, y\right) / d y$ and $\left.d F_{L}\left(x, Q^{2}, m^{2}, y\right) / d y\right)$ were already published in [2] for a range of $x$ values at fixed $Q^{2}$ and for a range of $Q^{2}$ values at fixed $x$. These ranges are covered by the $\mathrm{H} 1$ and ZEUS detectors in HERA. We have reproduced these plots with our new programs. Also, we have previously published distributions in the invariant mass of the $Q \bar{Q}$ pair, (i.e., $d F_{2}\left(x, Q^{2}, m^{2}, M\right) / d M$ and $\left.d F_{L}\left(x, Q^{2}, m^{2}, M\right) / d M\right)$ for charm production in [6]. Therefore we concentrate on new results involving distributions which are sensitive to the four momenta of all the final particles.

We start by considering the production of charm quarks. We use $m=m_{c}=$ $1.5 \mathrm{GeV} / \mathrm{c}^{2}$ and simply choose the factorization (renormalization) scale as $\mu^{2}=$ $Q^{2}+4\left(m_{c}^{2}+\left(P_{t}^{c}+P_{t}^{\bar{c}}\right)^{2} / 4\right)$. Note that there are many possible choices of scale as we have all components of the final four vectors. Aside from the $P_{t}$ dependence, this choice reduces to the usual choice of $\mu^{2}=Q^{2}$ for electroproduction of massless quarks and $\mu^{2}=4 m_{c}^{2}$ for the photoproduction of charm quarks. We introduce a $P_{t}$ dependence by adding in the average of the magnitude of the transverse momenta of the heavy quark and heavy antiquark. As mentioned earlier we use the CTEQ3M parton densities [7] in the $\overline{\mathrm{MS}}$ scheme and the two loop $\alpha_{s}$ with $\Lambda_{4}=0.239 \mathrm{GeV}$.

The first distribution we present basically measures the transverse momentum of the additional jet which recoils against the heavy quark pair. The $P_{t}$ distribution of the charm-anticharm pair is shown in fig. 8(a) where we plot $d F_{2}\left(x, Q^{2}, m_{c}^{2}, P_{t}\right) / d P_{t}$ as a function of $P_{t}$. The histograms are presented at fixed $Q^{2}=12(\mathrm{GeV} / c)^{2}$ for $x$ values of $4.2 \times 10^{-4}, 8.5 \times 10^{-4}, 1.6 \times 10^{-3}$ and $2.7 \times 10^{-3}$ respectively. One sees that the $P_{t}$ distribution peaks at small $P_{t}$ and has a small negative contribution in the lowest bin. This is a region where the dominant contribution is from counterevents so the weights can be negative. The results of this calculation require missing contributions from even higher order perturbation theory before this bin will have a positive weight. In general there will be corresponding bins in all the exclusive plots where the weights can be negative. The depth of the negative bins is a function of $x, Q^{2}$, and the choice of scale. Note that at larger $P_{t}$ the structure function is dominated by the contribution from the square of the bremsstrahlung graphs so the weights are positive. Figure $8(\mathrm{~b})$ shows the corresponding results for fixed $x=8.5 \times 10^{-4}$ plotted for the $Q^{2}$ values of $8.5(\mathrm{GeV} / c)^{2}, 12(\mathrm{GeV} / c)^{2}, 25(\mathrm{GeV} / c)^{2}$ and $50(\mathrm{GeV} / c)^{2}$ respectively. The distributions peak near small $P_{t}$ and are either small or negative in the first bin. The histograms with $Q^{2}=12(\mathrm{GeV} / c)^{2}$ and $x=8.5 \times 10^{-4}$ (the dotted line) are the same in figs. $8(\mathrm{a})$ and $8(\mathrm{~b})$. We have also used the same scales on the axes so one can easily see that there is a greater change if we fix $x$ and vary $Q^{2}$ than if we fix $Q^{2}$ and vary $x$. We will continue to use the same scale for all the pairs of later plots to simplify the comparison between them.

We now turn to the distributions in the azimuthal angle between the outgoing charm quark and charm antiquark which we denote as $\Delta \phi$. This is the angle between the $\mathbf{P}_{t}$ vectors of the heavy quark-antiquark in the c. m. frame of the virtualphoton-hadron system. Since we integrate over the azimuthal angle between the plane containing the incoming and outgoing leptons and the plane defined by the 
incoming parton and outgoing heavy quark (to limit our discussion to $F_{2}$ and $F_{L}$ ) we can only plot relative azimuthal correlations. In the Born approximation this distribution is a delta function at $\pi$, as their four momenta must balance. Due to the radiation of the additional light mass parton, the distribution has a tail extending below $\pi$ and has a valley at $\pi$. The distributions become negative in the highest bins. This negative region is a general feature of all exclusive calculations. Figure 9 (a) shows results for $d F_{2}\left(x, Q^{2}, m_{c}^{2}, \Delta \phi\right) / d(\Delta \phi)$ at the same values of fixed $Q^{2}$ and variable $x$ as chosen previously in fig. 8(a), while fig. 9 (b) shows the results for fixed $x$ and variable $Q^{2}$ as chosen previously in fig. $8(\mathrm{~b})$. Note again that the dotted histograms are the same in fig. 9 (a) and 9 (b), and there is more variation for fixed $x$ and changing $Q^{2}$ than for fixed $Q^{2}$ and changing $x$.

Finally we show distributions in the so-called heavy quark cone size variable $R=\sqrt{(\Delta \phi)^{2}+(\Delta \eta)^{2}}$ where $\Delta \phi$ is the azimuthal angle between the $\mathbf{P}_{t}$ vectors of charm-anticharm quarks in the c. $\mathrm{m}$. frame of the virtual-photon-hadron system and $\Delta \eta$ is the difference in pseudo-rapidities of the charm quark-antiquark pair. We define pseudo-rapidity to be $\eta=1 / 2 \ln [(1+\cos \theta) /(1-\cos \theta)]$ where $\theta$ is the angle the quark (antiquark) makes with the axis defined in the back-to-back photonhadron system. In fig. 10(a) we choose fixed $Q^{2}$ and variable $x$ as in fig. 8(a) and plot $d F_{2}\left(x, Q^{2}, m_{c}^{2}, R\right) / d R$. In fig. 10(b) we choose fixed $x$ and variable $Q^{2}$ as in fig. $8(\mathrm{~b})$. The influence of the additional radiation causes these distributions to peak below $R=\pi$ and develop a dip at values above $R=\pi$. The negative bins are unphysical and should be partially filled in by higher order corrections. The depth of the negative bins is a function of the bin width. If we use a wider bin, the dips will be less pronounced. Again, as in the previous cases, we note there is a greater change in the histograms when we fix $x$ and vary $Q^{2}$ than when we fix $Q^{2}$ and vary $x$.

The next six figures repeat the previous distributions but for $F_{L}\left(x, Q^{2}, m_{c}^{2}\right)$ rather than $F_{2}\left(x, Q^{2}, m_{c}^{2}\right)$. The general features are much the same, but the integrated results are smaller for $F_{L}\left(x, Q^{2}, m_{c}^{2}\right)$ than for $F_{2}\left(x, Q^{2}, m_{c}^{2}\right)$. Figures 11(a) and 11(b) show the distributions in the transverse momentum of the pair. Figures 12(a) and 12(b) show the distributions in the azimuthal angle between the $\mathbf{P}_{t}$ vectors of the outgoing charm quark and charm antiquark. Then we present in figs. 13(a) and 13(b) the histograms of the distributions in $R$.

For completeness we now repeat the last twelve distributions taking the heavy quark to be the bottom quark with mass $m_{b}=4.75 \mathrm{GeV} / c$ and the heavy-antiquark to be the bottom antiquark with the same mass. The renormalization (factorization ) scale is chosen to be $\mu^{2}=Q^{2}+m_{b}^{2}+\left(P_{t}^{b}+P_{t}^{\bar{b}}\right)^{2} / 4$. Aside from the $P_{t}$ dependence, this choice reduces to the usual choice of $\mu^{2}=Q^{2}$ for electroproduction of massless quarks and $\mu^{2}=m_{b}^{2}$ for the photoproduction of bottom quarks. Here we used the CTEQ3M ( $\overline{\mathrm{MS}}$ ) distributions and the two loop running coupling with $\Lambda_{5}=$ $0.158 \mathrm{GeV}$.

The integrated results are smaller for bottom quarks than for charm quarks reflecting a decrease in $F_{2}$ by a factor of approximately 50, and in $F_{L}$ by a factor of approximately 150. We refer the interested reader to [6] for tables containing $F_{2}\left(x, Q^{2}, m^{2}\right)$ and $F_{L}\left(x, Q^{2}, m^{2}\right)$ for both charm and bottom quarks in the same $x$ 
and $Q^{2}$ bins presented here. Apart from this decrease there are general features which are to be expected. In figs. 14(a), 14(b), 17(a), and 17(b) we see that the $P_{t}$ spectra are harder. The $\Delta \phi$ histograms in figs. 15(a), 15(b), 18(a), and 18(b) show dominant back-to-back peaking, which is stronger for bottom quarks than for charm quarks as the NLO corrections are correspondingly smaller. Finally in figs. 16(a), 16(b), 19(a), and 19(b) we see that the $R$ histograms are very $Q^{2}$ dependent.

In addition to checking that all plots presented are indeed independent of the parameters $\tilde{\rho}$ and $\omega$ we have studied the renormalization (factorization) scale dependence of the $P_{t}, \Delta \phi$, and $R$ distributions presented here and the $M$ distributions presented in [6]. To aid the discussion we present tables 1-4 containing the averages of $P_{t}, \Delta \phi, R$, and $M$ as functions of the renormalization (factorization) scale for charm production. The subscripts 2 and $L$ refer to averages over $F_{2}$ and $F_{L}$ respectively. As customary we have presented averages for the renormalization (factorization) scale $\mu=\mu_{0}$ chosen in the previous plots and for $\mu=\mu_{0} / 2$ and $\mu=2 \mu_{0}$. In tables $5-8$ we show the same quantities for bottom production. Typical scale variations from the central value are around 5 percent for charm production and 1 percent for bottom production. In general the shapes of the $P_{t}, \Delta \phi$, and $R$ distributions remain fixed while we vary the scale but the normalization changes. This is to be expected because the corresponding plots are delta functions at lowest order. However, the $M$ distributions have contributions from LO already so while the normalization stays roughly fixed the shape of the plot changes slightly reflecting the change in the average values in the tables.

Our programs can also produce two dimensional plots. For example one might study $d F_{k}\left(x, Q^{2}, m^{2}, y^{Q}, y^{\bar{Q}}\right) / d y^{Q} d y^{\bar{Q}}$ or $d F_{k}\left(x, Q^{2}, m^{2}, y^{Q}, M\right) / d y^{Q} d M$ or other combinations. In addition, experimental cuts can be implemented.

\section{Conclusion}

In this article we have outlined the NLO calculation of the virtual-photon-parton (Wilson) coefficient functions in the exclusive production of heavy quarks plus one jet. This completes the study of the NLO electroproduction of heavy quarks. The single particle inclusive calculation has already been published [1], [2]. Also there are fits available in [5] for the $\eta$ and $\xi$ dependence of the scale independent coefficient functions allowing for a fast numerical estimation of integrated rates for experiments. In this series of papers we have concentrated on deep-inelastic electroproduction where there is no need to introduce any partonic densities in the photon.

The computer program we have written for the exclusive calculation has the advantage that the four vectors of the heavy quark, heavy-antiquark and/or one additional light parton jet are produced for each event and can be subjected to experimental cuts. We have not done this in any of the plots shown here but the computer program is available and can be easily modified to incorporate acceptances of the detectors at HERA. T We have previously presented NLO distributions in the invariant masses of the heavy quark heavy-antiquark pair [6] and shown that they

\footnotetext{
${ }^{1}$ Requests for the computer program should be sent to smith@elsebeth.physics.sunysb.edu.
} 
have reasonably smooth $K$-factors, i.e., one can generate these distributions by multiplying the Born differential cross sections by constant factors, usually around $K=1.3$ for bottom and 1.7 for charm. However, the study [2] of the single particle inclusive distributions in $p_{t}$ and $y$ showed that they do not have smooth $K$ factors.

For this paper we have presented those plots which depend on information from the four vector of the additional jet. We showed the distributions in the transversemomentum $\left(P_{t}\right)$ of the heavy quark antiquark pair, in the azimuthal angle $(\Delta \phi)$ between the $\mathbf{P}_{t}$ vectors of the heavy quark and heavy antiquark, and in the distribution in the heavy quark cone size $(R)$. All quantities were predicted in the c. $m$. frame of the photon-proton system after integration over the azimuthal angle between the plane containing the incoming and outgoing lepton and the plane containing the incoming proton and outgoing heavy quark. The results were presented as distributions in $F_{2}\left(x, Q^{2}, m^{2}\right)$ and $F_{L}\left(x, Q^{2}, m^{2}\right)$ at specific points in $x, Q^{2}$ and $m^{2}=m_{c}^{2}$ or $m^{2}=m_{b}^{2}$. None of these distributions can be reproduced by any $K$-factor multiplication as the corresponding Born distributions are proportional to delta-functions. The spread of the tails in these distributions indirectly measures the relative size of the NLO contribution to that of the LO contribution. In all cases the histograms of these distributions have negative bins. These are regions where the NLO calculation is not sufficient and a NNLO order calculation (or some form of resummation) should be made. A general statement about the magnitude of the NLO contribution compared to the LO one is difficult to make as the size and sign of the corrections may vary strongly between different regions of phase space. However, we see that all plots have larger $Q^{2}$ variation at fixed $x$ as comparied to varying $x$ at fixed $Q^{2}$. By varying the renormalization (factorization) scale we observed that the distributions presented here changed in normalization but not in shape while the invariant mass distribution presented earlier [6] kept approximately the same normalization and had a mild shape change. In our study we also calculated averages of various quantities and found the typical variation between central and extreme scale choices of 5 percent for charm production and 1 percent for bottom production.

\section{Acknowledgments}

We acknowledge S. Mendoza for help in the early stages of the project. We also thank E. Laenen, S. Riemersma, W. L. van Neerven, and J. Whitmore for helpful discussions and Michael Fischer for maintaining our computers. Our research is supported in part by the contract NSF 9309888. 
Table 1

\begin{tabular}{||c|c||c|c|c||c|c|c||}
\hline \hline \multicolumn{2}{||c||}{ Range } & \multicolumn{3}{c||}{$\left\langle P_{t}\right\rangle_{2}$} & \multicolumn{3}{c||}{$\langle\Delta \phi\rangle_{2}$} \\
\hline \hline$x$ & $Q^{2}$ & $\mu=\mu_{0} / 2$ & $\mu=\mu_{0}$ & $\mu=2 \mu_{0}$ & $\mu=\mu_{0} / 2$ & $\mu=\mu_{0}$ & $\mu=2 \mu_{0}$ \\
\hline \hline $8.5 \times 10^{-4}$ & 8.5 & 2.73 & 1.82 & 1.35 & 1.45 & 2.01 & 2.32 \\
\hline $8.5 \times 10^{-4}$ & 12 & 3.02 & 2.04 & 1.51 & 1.42 & 1.97 & 2.28 \\
\hline $8.5 \times 10^{-4}$ & 25 & 3.72 & 2.59 & 1.95 & 1.39 & 1.91 & 2.22 \\
\hline $8.5 \times 10^{-4}$ & 50 & 4.53 & 3.24 & 2.48 & 1.40 & 1.88 & 2.17 \\
\hline & & & & & & & \\
\hline $4.2 \times 10^{-4}$ & 12 & 3.63 & 2.45 & 1.80 & 1.16 & 1.79 & 2.15 \\
\hline $8.5 \times 10^{-4}$ & 12 & 3.02 & 2.04 & 1.51 & 1.42 & 1.97 & 2.28 \\
\hline $1.6 \times 10^{-3}$ & 12 & 2.52 & 1.71 & 1.28 & 1.63 & 2.12 & 2.39 \\
\hline $2.7 \times 10^{-3}$ & 12 & 2.14 & 1.46 & 1.10 & 1.79 & 2.23 & 2.47 \\
\hline \hline
\end{tabular}

Table 1. Variation of $\left\langle P_{t}\right\rangle_{2}$ and $\langle\Delta \phi\rangle_{2}$ for charm production with $\mu_{0}^{2}=Q^{2}+4\left(m_{c}^{2}+\right.$ $\left.\left(P_{t}^{c}+P_{t}^{\bar{c}}\right)^{2} / 4\right)$ for various $x$ and $Q^{2}$ values. 
Table 2

\begin{tabular}{||c|c||c|c|c||c|c|c||}
\hline \hline \multicolumn{2}{|c||}{ Range } & \multicolumn{3}{c||}{$\langle R\rangle_{2}$} & \multicolumn{3}{c||}{$\langle M\rangle_{2}$} \\
\hline \hline$x$ & $Q^{2}$ & $\mu=\mu_{0} / 2$ & $\mu=\mu_{0}$ & $\mu=2 \mu_{0}$ & $\mu=\mu_{0} / 2$ & $\mu=\mu_{0}$ & $\mu=2 \mu_{0}$ \\
\hline \hline $8.5 \times 10^{-4}$ & 8.5 & 2.25 & 2.70 & 2.93 & 7.96 & 7.58 & 7.31 \\
\hline $8.5 \times 10^{-4}$ & 12 & 2.27 & 2.70 & 2.94 & 8.54 & 8.14 & 7.87 \\
\hline $8.5 \times 10^{-4}$ & 25 & 2.34 & 2.76 & 3.00 & 10.2 & 9.77 & 9.45 \\
\hline $8.5 \times 10^{-4}$ & 50 & 2.49 & 2.87 & 3.11 & 12.3 & 12.0 & 11.7 \\
\hline & & & & & & & \\
\hline $4.2 \times 10^{-4}$ & 12 & 2.05 & 2.55 & 2.83 & 8.98 & 8.54 & 8.21 \\
\hline $8.5 \times 10^{-4}$ & 12 & 2.27 & 2.70 & 2.94 & 8.54 & 8.14 & 7.87 \\
\hline $1.6 \times 10^{-3}$ & 12 & 2.45 & 2.83 & 3.03 & 8.09 & 7.75 & 7.51 \\
\hline $2.7 \times 10^{-3}$ & 12 & 2.59 & 2.92 & 3.10 & 7.72 & 7.41 & 7.21 \\
\hline \hline
\end{tabular}

Table 2. Variation of $\langle R\rangle_{2}$ and $\langle M\rangle_{2}$ for charm production with $\mu_{0}^{2}=Q^{2}+4\left(m_{c}^{2}+\right.$ $\left.\left(P_{t}^{c}+P_{t}^{\bar{c}}\right)^{2} / 4\right)$ for various $x$ and $Q^{2}$ values. 
Table 3

\begin{tabular}{||c|c||c|c|c||c|c|c||}
\hline \hline \multicolumn{2}{|c||}{ Range } & \multicolumn{3}{c||}{$\left\langle P_{t}\right\rangle_{L}$} & \multicolumn{3}{c||}{$\langle\Delta \phi\rangle_{L}$} \\
\hline \hline$x$ & $Q^{2}$ & $\mu=\mu_{0} / 2$ & $\mu=\mu_{0}$ & $\mu=2 \mu_{0}$ & $\mu=\mu_{0} / 2$ & $\mu=\mu_{0}$ & $\mu=2 \mu_{0}$ \\
\hline \hline $8.5 \times 10^{-4}$ & 8.5 & 2.47 & 1.73 & 1.31 & 2.17 & 2.45 & 2.62 \\
\hline $8.5 \times 10^{-4}$ & 12 & 2.78 & 1.96 & 1.50 & 2.14 & 2.42 & 2.59 \\
\hline $8.5 \times 10^{-4}$ & 25 & 3.58 & 2.60 & 2.00 & 2.12 & 2.38 & 2.55 \\
\hline $8.5 \times 10^{-4}$ & 50 & 4.60 & 3.44 & 2.68 & 2.13 & 2.36 & 2.53 \\
\hline & & & & & & & \\
\hline $4.2 \times 10^{-4}$ & 12 & 3.25 & 2.30 & 1.75 & 2.02 & 2.33 & 2.52 \\
\hline $8.5 \times 10^{-4}$ & 12 & 2.78 & 1.96 & 1.50 & 2.14 & 2.42 & 2.59 \\
\hline $1.6 \times 10^{-3}$ & 12 & 2.37 & 1.68 & 1.28 & 2.25 & 2.51 & 2.66 \\
\hline $2.7 \times 10^{-3}$ & 12 & 2.05 & 1.45 & 1.12 & 2.34 & 2.57 & 2.71 \\
\hline \hline
\end{tabular}

Table 3. Variation of $\left\langle P_{t}\right\rangle_{L}$ and $\langle\Delta \phi\rangle_{L}$ for charm production with $\mu_{0}^{2}=Q^{2}+4\left(m_{c}^{2}+\right.$ $\left.\left(P_{t}^{c}+P_{t}^{\bar{c}}\right)^{2} / 4\right)$ for various $x$ and $Q^{2}$ values. 
Table 4

\begin{tabular}{||c|c||c|c|c||c|c|c||}
\hline \hline \multicolumn{2}{||c||}{ Range } & \multicolumn{3}{c||}{$\langle R\rangle_{L}$} & \multicolumn{3}{c||}{$\langle M\rangle_{L}$} \\
\hline \hline$x$ & $Q^{2}$ & $\mu=\mu_{0} / 2$ & $\mu=\mu_{0}$ & $\mu=2 \mu_{0}$ & $\mu=\mu_{0} / 2$ & $\mu=\mu_{0}$ & $\mu=2 \mu_{0}$ \\
\hline \hline $8.5 \times 10^{-4}$ & 8.5 & 2.59 & 2.78 & 2.90 & 6.15 & 5.97 & 5.86 \\
\hline $8.5 \times 10^{-4}$ & 12 & 2.58 & 2.77 & 2.89 & 6.56 & 6.36 & 6.23 \\
\hline $8.5 \times 10^{-4}$ & 25 & 2.58 & 2.77 & 2.89 & 7.75 & 7.53 & 7.39 \\
\hline $8.5 \times 10^{-4}$ & 50 & 2.62 & 2.79 & 2.91 & 9.42 & 9.17 & 9.04 \\
\hline & & & & & & & \\
\hline $4.2 \times 10^{-4}$ & 12 & 2.01 & 2.70 & 2.84 & 6.80 & 6.51 & 6.34 \\
\hline $8.5 \times 10^{-4}$ & 12 & 2.58 & 2.77 & 2.89 & 6.56 & 6.63 & 6.23 \\
\hline $1.6 \times 10^{-3}$ & 12 & 2.65 & 2.83 & 2.94 & 6.39 & 6.21 & 6.11 \\
\hline $2.7 \times 10^{-3}$ & 12 & 2.71 & 2.87 & 2.97 & 6.21 & 6.09 & 5.99 \\
\hline \hline
\end{tabular}

Table 4. Variation of $\langle R\rangle_{L}$ and $\langle M\rangle_{L}$ for charm production with $\mu_{0}^{2}=Q^{2}+4\left(m_{c}^{2}+\right.$ $\left.\left(P_{t}^{c}+P_{t}^{\bar{c}}\right)^{2} / 4\right)$ for various $x$ and $Q^{2}$ values. 
Table 5

\begin{tabular}{||c|c||c|c|c||c|c|c||}
\hline \hline \multicolumn{2}{|c||}{ Range } & \multicolumn{3}{c||}{$\left\langle P_{t}\right\rangle_{2}$} & \multicolumn{3}{c||}{$\langle\Delta \phi\rangle_{2}$} \\
\hline \hline$x$ & $Q^{2}$ & $\mu=\mu_{0} / 2$ & $\mu=\mu_{0}$ & $\mu=2 \mu_{0}$ & $\mu=\mu_{0} / 2$ & $\mu=\mu_{0}$ & $\mu=2 \mu_{0}$ \\
\hline \hline $8.5 \times 10^{-4}$ & 8.5 & 3.52 & 2.54 & 1.94 & 2.29 & 2.52 & 2.66 \\
\hline $8.5 \times 10^{-4}$ & 12 & 3.88 & 2.83 & 2.17 & 2.24 & 2.48 & 2.63 \\
\hline $8.5 \times 10^{-4}$ & 25 & 4.64 & 3.47 & 2.70 & 2.17 & 2.41 & 2.56 \\
\hline $8.5 \times 10^{-4}$ & 50 & 5.36 & 4.11 & 3.25 & 2.13 & 2.35 & 2.51 \\
\hline & & & & & & & \\
\hline $4.2 \times 10^{-4}$ & 12 & 4.70 & 3.48 & 2.68 & 2.12 & 2.37 & 2.54 \\
\hline $8.5 \times 10^{-4}$ & 12 & 3.88 & 2.83 & 2.17 & 2.24 & 2.48 & 2.63 \\
\hline $1.6 \times 10^{-3}$ & 12 & 3.16 & 2.27 & 1.74 & 2.35 & 2.57 & 2.70 \\
\hline $2.7 \times 10^{-3}$ & 12 & 2.57 & 1.82 & 1.40 & 2.45 & 2.65 & 2.76 \\
\hline \hline
\end{tabular}

Table 5. Variation of $\left\langle P_{t}\right\rangle_{2}$ and $\langle\Delta \phi\rangle_{2}$ for bottom production with $\mu_{0}^{2}=Q^{2}+m_{b}^{2}+$ $\left(P_{t}^{b}+P_{t}^{\bar{b}}\right)^{2} / 4$ for various $x$ and $Q^{2}$ values. 
Table 6

\begin{tabular}{||c|c||c|c|c||c|c|c||}
\hline \hline \multicolumn{2}{|c||}{ Range } & \multicolumn{3}{c||}{$\langle R\rangle_{2}$} & \multicolumn{3}{c||}{$\langle M\rangle_{2}$} \\
\hline \hline$x$ & $Q^{2}$ & $\mu=\mu_{0} / 2$ & $\mu=\mu_{0}$ & $\mu=2 \mu_{0}$ & $\mu=\mu_{0} / 2$ & $\mu=\mu_{0}$ & $\mu=2 \mu_{0}$ \\
\hline \hline $8.5 \times 10^{-4}$ & 8.5 & 2.77 & 2.96 & 3.07 & 16.7 & 16.6 & 16.4 \\
\hline $8.5 \times 10^{-4}$ & 12 & 2.74 & 2.93 & 3.05 & 17.3 & 17.2 & 17.0 \\
\hline $8.5 \times 10^{-4}$ & 25 & 2.71 & 2.89 & 3.02 & 18.8 & 18.6 & 18.4 \\
\hline $8.5 \times 10^{-4}$ & 50 & 2.71 & 2.88 & 3.01 & 20.5 & 20.3 & 20.1 \\
\hline & & & & & & & \\
\hline $4.2 \times 10^{-4}$ & 12 & 2.64 & 2.85 & 2.98 & 18.3 & 18.1 & 17.8 \\
\hline $8.5 \times 10^{-4}$ & 12 & 2.74 & 2.93 & 3.05 & 17.3 & 17.2 & 17.0 \\
\hline $1.6 \times 10^{-3}$ & 12 & 2.83 & 3.00 & 3.11 & 16.4 & 16.3 & 16.1 \\
\hline $2.7 \times 10^{-3}$ & 12 & 2.90 & 3.06 & 3.15 & 15.6 & 15.5 & 15.4 \\
\hline \hline
\end{tabular}

Table 6. Variation of $\langle R\rangle_{2}$ and $\langle M\rangle_{2}$ for bottom production with $\mu_{0}^{2}=Q^{2}+m_{b}^{2}+$ $\left(P_{t}^{b}+P_{t}^{\bar{b}}\right)^{2} / 4$ for various $x$ and $Q^{2}$ values. 
Table 7

\begin{tabular}{||c|c||c|c|c||c|c|c||}
\hline \hline \multicolumn{2}{|c||}{ Range } & \multicolumn{3}{c||}{$\left\langle P_{t}\right\rangle_{L}$} & \multicolumn{3}{c||}{$\langle\Delta \phi\rangle_{L}$} \\
\hline \hline$x$ & $Q^{2}$ & $\mu=\mu_{0} / 2$ & $\mu=\mu_{0}$ & $\mu=2 \mu_{0}$ & $\mu=\mu_{0} / 2$ & $\mu=\mu_{0}$ & $\mu=2 \mu_{0}$ \\
\hline \hline $8.5 \times 10^{-4}$ & 8.5 & 2.89 & 2.24 & 1.80 & 2.65 & 2.75 & 2.82 \\
\hline $8.5 \times 10^{-4}$ & 12 & 3.29 & 2.54 & 2.03 & 2.62 & 2.72 & 2.80 \\
\hline $8.5 \times 10^{-4}$ & 25 & 4.08 & 3.18 & 2.55 & 2.56 & 2.68 & 2.76 \\
\hline $8.5 \times 10^{-4}$ & 50 & 4.86 & 3.85 & 3.13 & 2.52 & 2.64 & 2.73 \\
\hline & & & & & & & \\
\hline $4.2 \times 10^{-4}$ & 12 & 3.91 & 3.05 & 2.46 & 2.55 & 2.67 & 2.75 \\
\hline $8.5 \times 10^{-4}$ & 12 & 3.29 & 2.54 & 2.03 & 2.62 & 2.72 & 2.80 \\
\hline $1.6 \times 10^{-3}$ & 12 & 2.72 & 2.08 & 1.67 & 2.67 & 2.77 & 2.84 \\
\hline $2.7 \times 10^{-3}$ & 12 & 2.25 & 1.72 & 1.38 & 2.72 & 2.81 & 2.87 \\
\hline \hline
\end{tabular}

Table 7. Variation of $\left\langle P_{t}\right\rangle_{L}$ and $\langle\Delta \phi\rangle_{L}$ for bottom production with $\mu_{0}^{2}=Q^{2}+m_{b}^{2}+$ $\left(P_{t}^{b}+P_{t}^{\bar{b}}\right)^{2} / 4$ for various $x$ and $Q^{2}$ values. 
Table 8

\begin{tabular}{||c|c||c|c|c||c|c|c||}
\hline \hline \multicolumn{2}{||c||}{ Range } & \multicolumn{3}{c||}{$\langle R\rangle_{L}$} & \multicolumn{3}{c||}{$\langle M\rangle_{L}$} \\
\hline \hline$x$ & $Q^{2}$ & $\mu=\mu_{0} / 2$ & $\mu=\mu_{0}$ & $\mu=2 \mu_{0}$ & $\mu=\mu_{0} / 2$ & $\mu=\mu_{0}$ & $\mu=2 \mu_{0}$ \\
\hline \hline $8.5 \times 10^{-4}$ & 8.5 & 2.91 & 2.98 & 3.02 & 15.0 & 14.9 & 14.8 \\
\hline $8.5 \times 10^{-4}$ & 12 & 2.88 & 2.95 & 3.00 & 15.3 & 15.1 & 15.0 \\
\hline $8.5 \times 10^{-4}$ & 25 & 2.84 & 2.92 & 2.98 & 16.0 & 15.9 & 15.8 \\
\hline $8.5 \times 10^{-4}$ & 50 & 2.82 & 2.90 & 2.96 & 17.1 & 17.0 & 16.8 \\
\hline & & & & & & & \\
\hline $4.2 \times 10^{-4}$ & 12 & 2.84 & 2.91 & 2.97 & 15.7 & 15.5 & 15.3 \\
\hline $8.5 \times 10^{-4}$ & 12 & 2.88 & 2.95 & 3.00 & 15.3 & 15.1 & 15.0 \\
\hline $1.6 \times 10^{-3}$ & 12 & 2.92 & 2.99 & 3.04 & 14.8 & 14.8 & 14.7 \\
\hline $2.7 \times 10^{-3}$ & 12 & 2.96 & 3.02 & 3.06 & 14.4 & 14.4 & 14.3 \\
\hline \hline
\end{tabular}

Table 8. Variation of $\langle R\rangle_{L}$ and $\langle M\rangle_{L}$ for bottom production with $\mu_{0}^{2}=Q^{2}+m_{b}^{2}+$ $\left(P_{t}^{b}+P_{t}^{\bar{b}}\right)^{2} / 4$ for various $x$ and $Q^{2}$ values. 


\section{Appendix A}

Here we discuss the kinematic variables and phase space in the $2 \rightarrow 2$ and $2 \rightarrow 3$ reactions. We then define the generalized plus distributions used in the text. The Mandelstam invariants for the reaction,

$$
\gamma^{*}(q)+a_{1}\left(k_{1}\right) \rightarrow Q\left(p_{1}\right)+\bar{Q}\left(p_{2}\right),
$$

with $k_{1}^{2}=0$ and $p_{i}^{2}=m^{2}$ are

$$
\begin{aligned}
s^{\prime} & \equiv s-q^{2}=\left(q+k_{1}\right)^{2}-q^{2}=2 q \cdot k_{1}, \\
t_{1} & \equiv t-m^{2}=\left(k_{1}-p_{2}\right)^{2}-m^{2}=-2 k_{1} \cdot p_{2}, \\
u_{1} & \equiv u-m^{2}=\left(q-p_{2}\right)^{2}-m^{2}=-2 q \cdot p_{2}+q^{2},
\end{aligned}
$$

which satisfy $s^{\prime}+t_{1}+u_{1}=0$. The two body phase space in space-time dimension $n=4+\epsilon$ is

$$
d \Gamma_{2}=\frac{2^{-\epsilon}}{16 \pi}\left(\frac{s}{4 \pi}\right)^{\epsilon / 2} \beta^{1+\epsilon} \frac{1}{\Gamma(1+\epsilon / 2)} \sin ^{1+\epsilon} \theta_{1} d \theta_{1}
$$

where $\beta=\sqrt{1-\rho}, \rho=4 m^{2} / s$ and $\theta_{1}$ is the angle between $\mathbf{q}$ and $\mathbf{p}_{1}$ in the $\gamma^{*} a_{1}$ center-of-mass frame. Therefore we have

$$
\begin{aligned}
t_{1} & =-\frac{1}{2} s^{\prime}\left(1-\beta \cos \theta_{1}\right), \\
u_{1} & =-\frac{1}{2} s^{\prime}\left(1+\beta \cos \theta_{1}\right),
\end{aligned}
$$

with $0 \leq \theta_{1} \leq \pi$.

For the two to three body process

$$
\gamma^{*}(q)+a_{1}\left(k_{1}\right) \rightarrow Q\left(p_{1}\right)+\bar{Q}\left(p_{2}\right)+a_{2}\left(k_{2}\right),
$$

with $k_{i}^{2}=0$ and $p_{i}^{2}=m^{2}$ there are 5 independent invariants which we take to be

$$
\begin{aligned}
s^{\prime} & =s-q^{2}=\left(q+k_{1}\right)^{2}-q^{2}=2 q \cdot k_{1}, \\
t_{1} & =\left(k_{1}-p_{2}\right)^{2}-m^{2}=-2 k_{1} \cdot p_{2}, \\
u_{1}^{\prime} & \equiv u_{1}-q^{2}=\left(q-p_{2}\right)^{2}-m^{2}-q^{2}=-2 q \cdot p_{2}, \\
t^{\prime} & =\left(k_{1}-k_{2}\right)^{2}=-2 k_{1} \cdot k_{2}, \\
u^{\prime} & =\left(q-k_{2}\right)^{2}=q^{2}-2 q \cdot k_{2},
\end{aligned}
$$

where $q+k_{1}=k_{2}+p_{1}+p_{2}$. The remaining five invariants are

$$
\begin{aligned}
& s_{3}=\left(k_{2}+p_{2}\right)^{2}-m^{2}=-s^{\prime}-u^{\prime}-t^{\prime}-u_{1}^{\prime}-t_{1}=2 k_{2} \cdot p_{2}, \\
& s_{4}=\left(k_{2}+p_{1}\right)^{2}-m^{2}=s^{\prime}+u_{1}+t_{1}=2 k_{2} \cdot p_{1}, \\
& s_{5}=\left(p_{1}+p_{2}\right)^{2}=s^{\prime}+u^{\prime}+t^{\prime}=2 m^{2}+2 p_{1} \cdot p_{2}, \\
& u_{6}=\left(k_{1}-p_{1}\right)^{2}-m^{2}=-s^{\prime}-t^{\prime}-t_{1}=-2 k_{1} \cdot p_{1}, \\
& u_{7}=\left(q-p_{1}\right)^{2}-m^{2}=-s^{\prime}-u^{\prime}-u_{1}^{\prime}=q^{2}-2 p_{1} \cdot q .
\end{aligned}
$$


We introduce the variables $x=s_{5}^{\prime} / s^{\prime}$ where $s_{5}^{\prime}=s_{5}-q^{2}$, and $y$ is the cosine of the angle between $\mathbf{q}$ and $\mathbf{k}_{2}$ in the $\gamma^{*} a_{1}$ center-of-mass frame. These have ranges $\rho^{*} \leq x \leq 1$ and $-1 \leq y \leq 1$ with $\rho^{*}=\left(4 m^{2}-q^{2}\right) / s^{\prime}$. We then find

$$
\begin{aligned}
t^{\prime} & =-\frac{1}{2} s^{\prime}\left(\frac{s^{\prime}}{s}\right)(1-x)(1+y), \\
u^{\prime} & =q^{2}\left(\frac{s_{5}}{s}\right)-\frac{1}{2} s^{\prime}\left(\frac{s^{\prime}}{s}\right)(1-x)(1-y) .
\end{aligned}
$$

In the center-of-mass system of the outgoing heavy quark antiquark pair we decompose the momenta as follows:

$$
\begin{aligned}
q & =\left(q^{0}, 0,0,|\mathbf{q}|\right) \\
k_{1} & =k_{1}^{0}(1,0, \sin \psi, \cos \psi) \\
k_{2} & =\left(k_{2}^{0}, 0, k_{1}^{0} \sin \psi,|\mathbf{q}|+k_{1}^{0} \cos \psi\right) \\
p_{1} & =\frac{1}{2} \sqrt{s_{5}}\left(1, \beta_{5} \sin \theta_{2} \sin \theta_{1}, \beta_{5} \cos \theta_{2} \sin \theta_{1}, \beta_{5} \cos \theta_{1}\right), \\
p_{2} & =\frac{1}{2} \sqrt{s_{5}}\left(1,-\beta_{5} \sin \theta_{2} \sin \theta_{1},-\beta_{5} \cos \theta_{2} \sin \theta_{1},-\beta_{5} \cos \theta_{1}\right)
\end{aligned}
$$

where

$$
\begin{aligned}
q^{0} & =\frac{s+u^{\prime}}{2 \sqrt{s_{5}}} \\
|\mathbf{q}| & =\frac{1}{2 \sqrt{s_{5}}} \sqrt{\left(s+u^{\prime}\right)^{2}-4 s_{5} q^{2}} \\
k_{1}^{0} & =\frac{s_{5}-u^{\prime}}{2 \sqrt{s_{5}}} \\
k_{2}^{0} & =\frac{s-s_{5}}{2 \sqrt{s_{5}}} \\
\cos \psi & =\frac{-s^{\prime}+2 k_{1}^{0} q^{0}}{2 k_{1}^{0}|\mathbf{q}|} \\
\beta_{5} & =\sqrt{1-4 m^{2} / s_{5}} .
\end{aligned}
$$

Note that in the limit $x \rightarrow 1, \beta_{5} \rightarrow \beta$. The remaining two independent invariants are

$$
\begin{aligned}
t_{1} & =-\frac{1}{2}\left(s_{5}-u^{\prime}\right)\left(1+\beta_{5} \cos \theta_{2} \sin \theta_{1} \sin \psi+\beta_{5} \cos \theta_{1} \cos \psi\right), \\
u_{1} & =q^{2}-\frac{1}{2}\left(s+u^{\prime}+2 \sqrt{s_{5}} \beta_{5}|\mathbf{q}| \cos \theta_{1}\right) .
\end{aligned}
$$

We will also need the following expressions

$$
\begin{aligned}
& s_{3}=\frac{1}{2}\left[s-s_{5}+\left(s_{5}-u^{\prime}\right) \beta_{5} \cos \theta_{2} \sin \theta_{1} \sin \psi+\beta_{5} \cos \theta_{1}\left(2 \sqrt{s_{5}}|\mathbf{q}|+\left(s_{5}-u^{\prime}\right) \cos \psi\right)\right], \\
& s_{4}=\frac{1}{2}\left[s-s_{5}-\left(s_{5}-u^{\prime}\right) \beta_{5} \cos \theta_{2} \sin \theta_{1} \sin \psi-\beta_{5} \cos \theta_{1}\left(2 \sqrt{s_{5}}|\mathbf{q}|+\left(s_{5}-u^{\prime}\right) \cos \psi\right)\right] .
\end{aligned}
$$


The three body phase space in $n=4+\epsilon$ space-time dimensions expressed in terms of $x, y, \theta_{1}$, and $\theta_{2}$ is

$$
d \Gamma_{3}=H N d \Gamma_{2}^{(5)} \frac{\left(s^{\prime}\right)^{1+\epsilon / 2}}{2 \pi}\left(\frac{s^{\prime}}{s}\right)^{1+\epsilon / 2}(1-x)^{1+\epsilon}\left(1-y^{2}\right)^{\epsilon / 2} d y \sin ^{\epsilon} \theta_{2} d \theta_{2},
$$

with $0 \leq \theta_{1} \leq \pi, 0 \leq \theta_{2} \leq \pi, \rho^{*} \leq x \leq 1,-1 \leq y \leq 1$, and

$$
\begin{aligned}
& H=\frac{\Gamma(1+\epsilon / 2)}{\Gamma(1-\epsilon / 2) \Gamma(1+\epsilon)}=1-\frac{\pi^{2}}{12} \epsilon^{2}+O\left(\epsilon^{3}\right), \\
& N=\frac{(4 \pi)^{-\epsilon / 2}}{(4 \pi)^{2}} \Gamma(1-\epsilon / 2)=\frac{1}{16 \pi^{2}}\left(\frac{\epsilon}{2}\right)\left(\frac{2}{\bar{\epsilon}}\right)+O\left(\epsilon^{2}\right), \\
& d \Gamma_{2}^{(5)}=\frac{2^{-\epsilon}}{16 \pi}\left(\frac{s_{5}}{4 \pi}\right)^{\epsilon / 2} \beta_{5}^{1+\epsilon} \frac{1}{\Gamma(1+\epsilon / 2)} \sin ^{1+\epsilon} \theta_{1} d \theta_{1} d x,
\end{aligned}
$$

where $2 / \bar{\epsilon}=2 / \epsilon+\gamma_{E}-\ln 4 \pi$.

The generalized plus distributions encountered in sec. 3 arise by making the replacements

$$
\begin{aligned}
(1+y)^{-1+\epsilon} & \sim\left(\frac{1}{1+y}\right)_{\omega}+\delta(1+y)\left(\frac{1}{\epsilon}+\ln \omega\right)+O(\epsilon), \\
\left(1-y^{2}\right)^{-1+\epsilon} & \sim \frac{1}{2}\left[\left(\frac{1}{1+y}\right)_{\omega}+\left(\frac{1}{1-y}\right)_{\omega}\right] \\
+ & {[\delta(1+y)+\delta(1-y)]\left(\frac{1}{2 \epsilon}+\frac{1}{2} \ln 2 \omega\right)+O(\epsilon), } \\
(1-x)^{-1+\epsilon} & \sim\left(\frac{1}{1-x}\right)_{\tilde{\rho}}+\epsilon\left(\frac{\ln (1-x)}{1-x}\right)_{\tilde{\rho}}\left[\frac{1}{\epsilon}+2 \ln \tilde{\beta}+2 \epsilon \ln ^{2} \tilde{\beta}\right]+O\left(\epsilon^{2}\right),
\end{aligned}
$$

inside integrations over smooth functions. We have defined $\tilde{\beta}=\sqrt{1-\tilde{\rho}}$, and the generalized plus distributions are defined by

$$
\begin{aligned}
\int_{\tilde{\rho}}^{1} d x f(x)\left(\frac{1}{1-x}\right)_{\tilde{\rho}} & =\int_{\tilde{\rho}}^{1} d x \frac{f(x)-f(1)}{1-x}, \\
\int_{\tilde{\rho}}^{1} d x f(x)\left(\frac{\ln (1-x)}{1-x}\right)_{\tilde{\rho}} & =\int_{\tilde{\rho}}^{1} d x \frac{f(x)-f(1)}{1-x} \ln (1-x), \\
\int_{-1}^{-1+\omega} d y f(y)\left(\frac{1}{1+y}\right)_{\omega} & =\int_{-1}^{-1+\omega} d y \frac{f(y)-f(-1)}{1+y}, \\
\int_{1-\omega}^{1} d y f(y)\left(\frac{1}{1-y}\right)_{\omega} & =\int_{1-\omega}^{1} d y \frac{f(y)-f(1)}{1-y}
\end{aligned}
$$

where $\rho^{*} \leq \tilde{\rho}<1$ and $0<\omega \leq 2$. Thus we see, in distinction from the phase space slicing method, that the soft and collinear parameters $\tilde{\rho}$ and $\omega$ are not required to be small. It is understood that when the integration range does not enclose a singularity, the distribution sign is dropped (c.f. (3.44)). 


\section{Appendix B}

In this appendix we discuss the derivation of the soft limit $x=1$ of the matrix element $M_{i}^{g}(3)$. We use standard techniques for the emission of soft gluons [28], [19], [18].

Consider the diagram shown in fig. 3(d). The momenta are defined according to fig. 1. Take the incoming gluon to have color index $a$ and Lorentz index $\mu$, the outgoing gluon to have color index $c$ and Lorentz index $\sigma$, and the photon to have Lorentz index $\rho$. Using standard Feynman rules one finds

$$
I=-i g f^{a b c} V_{\mu \sigma \alpha}\left(k_{1},-k_{2}, k_{2}-k_{1}\right) \frac{\epsilon^{\mu}\left(k_{1}\right)}{2 k_{1} \cdot k_{2}} B^{b \alpha \rho}\left(q, k_{1}-k_{2}\right),
$$

where

$$
V_{\mu \sigma \alpha}\left(k_{1},-k_{2}, k_{2}-k_{1}\right)=\left(k_{1}+k_{2}\right)_{\alpha} g_{\mu \sigma}+\left(k_{1}-2 k_{2}\right)_{\mu} g_{\sigma \alpha}+\left(k_{2}-2 k_{1}\right)_{\sigma} g_{\mu \alpha} .
$$

$B_{i j}^{b \alpha \beta}$ is the sum of the lowest order diagrams in fig. 2 and we have chosen the Feynman gauge. Now by using current conservation $\left[\left(k_{1}-k_{2}\right)_{\alpha} B^{b \alpha \rho}\left(q, k_{1}-k_{2}\right)=0\right]$, and the transversality of the gluon polarization tensor $\left[\epsilon_{\mu}\left(k_{1}\right) k_{1}^{\mu}=0\right]$, and recalling that in the soft limit $k_{2} \rightarrow 0$, a short computation shows that

$$
I=i g f^{a b c} \frac{k_{1}^{\sigma}}{k_{1} \cdot k_{2}} \epsilon_{\mu}\left(k_{1}\right) B^{b \mu \rho}\left(q, k_{1}\right)+\text { finite terms as } k_{2} \rightarrow 0 .
$$

As we work in the Feynman gauge we must consider the interference between all diagrams in fig. 3 [32]. Analyzing them as we have fig. 3(d) leads to

$$
M_{i}^{g}(3)=8 g^{4} e^{2} e_{H}^{2} N C_{F}\left[C_{A} S_{i, O K}+2 C_{F} S_{i, Q E D}\right]
$$

in the soft limit when one neglects terms that are finite when multiplied by the $(1-x)^{1+\epsilon}$ term in the three body phase space. We have defined

$$
\begin{aligned}
S_{i, O K} & =\left[\left(k_{1} p_{2}\right)+\left(p_{1} k_{1}\right)-\left(p_{1} p_{2}\right)\right] B_{i, Q E D}, \\
S_{i, Q E D} & =\left[\left(p_{1} p_{2}\right)-\frac{1}{2}\left(p_{1} p_{1}\right)-\frac{1}{2}\left(p_{2} p_{2}\right)\right] B_{i, Q E D} .
\end{aligned}
$$

and denoted $(u v)=u \cdot v /\left(u \cdot k_{2} v \cdot k_{2}\right)$ which is often called the eikonal factor. Expanding the factors $(u v)$ we reproduce eqs. (3.21) and (3.22) of [1]. Recalling the definition of $f_{i}^{g}\left(\theta_{1}\right)$ we find

$$
\begin{aligned}
f_{i}^{g}\left(\theta_{1}\right) & =\left[\frac{1}{4}\left(s^{\prime}\right)^{2}\left(\frac{s^{\prime}}{s}\right)^{2}\right]\left[8 g^{4} e^{2} e_{H}^{2} N C_{F}\right] B_{i, Q E D} \\
& \times\left\{C_{A}\left[I_{\left(k_{1} p_{2}\right)}+I_{\left(p_{1} k_{1}\right)}-I_{\left(p_{1} p_{2}\right)}\right]+2 C_{F}\left[I_{\left(p_{1} p_{2}\right)}-\frac{1}{2} I_{\left(p_{1} p_{1}\right)}-\frac{1}{2} I_{\left(p_{2} p_{2}\right)}\right]\right\},
\end{aligned}
$$

with

$$
\left.I_{(u v)} \equiv \int_{-1}^{1}\left(1-y^{2}\right)^{-1+\epsilon / 2} d y \int_{0}^{\pi} \sin ^{\epsilon} \theta_{2} d \theta_{2}\left[(u v)(1-x)^{2}\left(1-y^{2}\right)\right]\right|_{x=1} .
$$


Using the expansion of $s_{3}$ and $s_{4}$ about $x=1$

$$
\begin{aligned}
& s_{3}=\frac{1}{2} s^{\prime}(1-x)\left[1+\beta \sqrt{1-y^{2}} \cos \theta_{2} \sin \theta_{1}+\beta y \cos \theta_{1}\right]+O\left((1-x)^{2}\right), \\
& s_{4}=\frac{1}{2} s^{\prime}(1-x)\left[1-\beta \sqrt{1-y^{2}} \cos \theta_{2} \sin \theta_{1}-\beta y \cos \theta_{1}\right]+O\left((1-x)^{2}\right),
\end{aligned}
$$

we can write the integrals $I_{(u v)}$ in terms of

$$
I_{n}^{(j)} \equiv \int_{0}^{\pi} d \alpha(\sin \alpha)^{n-3} \int_{0}^{\pi} d \beta(\sin \beta)^{n-4}(A+B \cos \alpha+C \sin \alpha \cos \beta)^{-j},
$$

with $j=1,2$ and $A^{2} \neq B^{2}+C^{2}$, and

$\bar{I}_{n}^{(i, j)} \equiv \int_{0}^{\pi} d \alpha(\sin \alpha)^{n-3} \int_{0}^{\pi} d \beta(\sin \beta)^{n-4}(A+B \cos \alpha+C \sin \alpha \cos \beta)^{-j}(a+b \cos \alpha)^{-i}$,

with $A^{2} \neq B^{2}+C^{2}, a=-b$, and $i=j=1$. The second integral may be found in the literature [29] and the first evaluated using the methods of [30]. We find

$$
\begin{aligned}
I_{n}^{(1)=} & \frac{\pi}{\sqrt{B^{2}+C^{2}}}\left\{\ln \left(\frac{A+\sqrt{B^{2}+C^{2}}}{A-\sqrt{B^{2}+C^{2}}}\right)\right. \\
& \left.-(n-4)\left[\operatorname{Li}_{2}\left(\frac{2 \sqrt{B^{2}+C^{2}}}{A+\sqrt{B^{2}+C^{2}}}\right)+\frac{1}{4} \ln ^{2}\left(\frac{A+\sqrt{B^{2}+C^{2}}}{A-\sqrt{B^{2}+C^{2}}}\right)\right]\right\},
\end{aligned}
$$

and

$$
I_{n}^{(2)}=\frac{2 \pi}{A^{2}-B^{2}-C^{2}}\left[1-\frac{1}{2}(n-4) \frac{A}{\sqrt{B^{2}+C^{2}}} \ln \left(\frac{A+\sqrt{B^{2}+C^{2}}}{A-\sqrt{B^{2}+C^{2}}}\right)\right],
$$

where we drop $O\left((n-4)^{2}\right)$ terms and quote, for completeness, the result for the second integral 29.

$$
\begin{aligned}
\bar{I}_{n}^{(1,1)} & =\frac{\pi}{a(A+B)}\left\{\frac{2}{n-4}+\ln \left(\frac{(A+B)^{2}}{A^{2}-B^{2}-C^{2}}\right)\right. \\
& +\frac{1}{2}(n-4)\left[\ln ^{2}\left(\frac{A-\sqrt{B^{2}+C^{2}}}{A+B}\right)-\frac{1}{2} \ln ^{2}\left(\frac{A+\sqrt{B^{2}+C^{2}}}{A-\sqrt{B^{2}+C^{2}}}\right)\right. \\
& \left.\left.+2 \operatorname{Li}_{2}\left(-\frac{B+\sqrt{B^{2}+C^{2}}}{A-\sqrt{B^{2}+C^{2}}}\right)-2 \operatorname{Li}_{2}\left(\frac{B-\sqrt{B^{2}+C^{2}}}{A+B}\right)\right]\right\},
\end{aligned}
$$

again dropping $O\left((n-4)^{2}\right)$ terms. The dilogarithmic function $\operatorname{Li}_{2}(x)$ is defined in [31].

From these integrals and $(\overline{B .4}),(B .5),(B .6)$, and $(3.8)$ we find

$$
d \sigma_{i, g}^{(s)}=C_{i, g} M_{i}^{s o f t} d \Gamma_{2}
$$


with

$$
M_{i}^{\text {soft }}=8 g^{4} e^{2} e_{H}^{2} N \mu^{-\epsilon} C_{\epsilon} C_{F}\left[C_{A} \tilde{S}_{i, O K}+2 C_{F} \tilde{S}_{i, Q E D}\right] B_{i, Q E D} .
$$

The factor

$$
C_{\epsilon}=\frac{1}{16 \pi^{2}} e^{\epsilon\left(\gamma_{E}-\ln 4 \pi\right) / 2}\left(\frac{\mu^{2}}{m^{2}}\right)^{-\epsilon / 2}
$$

is common not only to $d \sigma_{i, g}^{(s)}$ but to $d \sigma_{i, g}^{(v)}$ as well (see Appendix A of [1] ). The mass parameter $\mu$ originates from the dimensionality of the gauge coupling in $n$ dimensions. The remaining terms are

$$
\begin{aligned}
\tilde{S}_{i, O K} & =\frac{8}{\epsilon^{2}}-\frac{4}{\epsilon}\left[\frac{1}{\beta}\left(\frac{2 m^{2}}{s}-1\right) \ln \frac{1-\beta}{1+\beta}-4 \ln \tilde{\beta}-\ln \frac{-t_{1}}{m^{2}}-\ln \frac{-u_{1}}{m^{2}}\right] \\
& -\left[\ln \frac{s}{m^{2}}+2 \ln \frac{-t_{1}}{m^{2}}+2 \ln \frac{-u_{1}}{m^{2}}-4 \ln \frac{s^{\prime}}{m^{2}}\right] \ln \frac{s}{m^{2}} \\
& +4\left[\ln \frac{-t_{1}}{m^{2}}+\ln \frac{-u_{1}}{m^{2}}-\ln \frac{s^{\prime}}{m^{2}}\right] \ln \frac{s^{\prime}}{m^{2}}-\frac{1}{\beta}\left(\frac{2 m^{2}}{s}-1\right) \\
& \times\left\{\left[8 \ln \tilde{\beta}-2 \ln \frac{s}{m^{2}}+4 \ln \frac{s^{\prime}}{m^{2}}+\ln \frac{1-\beta}{1+\beta}\right] \ln \frac{1-\beta}{1+\beta}+4 \operatorname{Li}_{2}\left(\frac{2 \beta}{1+\beta}\right)\right\} \\
& +8\left(\ln \frac{-t_{1}}{m^{2}}+\ln \frac{-u_{1}}{m^{2}}\right) \ln \tilde{\beta}+16 \ln { }^{2} \tilde{\beta}-3 \zeta(2)-\ln ^{2} \frac{1-\beta}{1+\beta} \\
& +2 \operatorname{Li}_{2}\left(1+\frac{2 t_{1}}{s^{\prime}(1-\beta)}\right)-2 \operatorname{Li}_{2}\left(1+\frac{s^{\prime}}{2 t_{1}}(1+\beta)\right) \\
& +2 \operatorname{Li}_{2}\left(1+\frac{2 u_{1}}{s^{\prime}(1-\beta)}\right)-2 \operatorname{Li}_{2}\left(1+\frac{s^{\prime}}{2 u_{1}}(1+\beta)\right) \\
& +\ln ^{2}\left[-\frac{s^{\prime}}{2 t_{1}}(1-\beta)\right]+\ln ^{2}\left[-\frac{s^{\prime}}{2 u_{1}}(1-\beta)\right],
\end{aligned}
$$

and

$$
\begin{aligned}
\tilde{S}_{i, Q E D} & =\frac{4}{\epsilon}\left[\frac{1}{\beta}\left(\frac{2 m^{2}}{s}-1\right) \ln \frac{1-\beta}{1+\beta}-1\right]+\frac{1}{\beta}\left(\frac{2 m^{2}}{s}-1\right) \\
& \times\left\{\left[8 \ln \tilde{\beta}-2 \ln \frac{s}{m^{2}}+4 \ln \frac{s^{\prime}}{m^{2}}+\ln \frac{1-\beta}{1+\beta}\right] \ln \frac{1-\beta}{1+\beta}+4 \operatorname{Li}_{2}\left(\frac{2 \beta}{1+\beta}\right)\right\} \\
& -8 \ln \tilde{\beta}+2 \ln \frac{s}{m^{2}}-4 \ln \frac{s^{\prime}}{m^{2}}-\frac{2}{\beta} \ln \frac{1-\beta}{1+\beta},
\end{aligned}
$$

where $\zeta(2)=\pi^{2} / 6$. 


\section{Appendix C}

In this appendix we discuss the derivation of the collinear limit $y=-1$ of the matrix element $M_{i}^{g}(3)$. A similar analysis follows for $M_{i}^{q}(3)$ for which we simply quote the results at the end. We closely follow appendix B of [19] thereby deriving $f_{i}^{g}\left(x,-1, \theta_{1}, \theta_{2}\right)$ in $n$ dimensions (see also appendix $\mathrm{C}$ of [18]).

Consider the diagrams shown in fig. 3. The momenta are defined according to fig. 1. Take the incoming gluon to have color index $a$ and Lorentz index $\mu$, the outgoing gluon to have color index $c$ and Lorentz index $\sigma$, and the photon to have Lorentz index $\rho$. Then the sum of the diagrams is

$$
M^{a c \rho}=\epsilon^{\mu}\left(k_{1}\right) \bar{\epsilon}^{\sigma}\left(k_{2}\right)\left[D_{\mu \sigma}^{a c \rho}+R_{\mu \sigma}^{a c \rho}\right],
$$

where $D_{\mu \sigma}^{a c \rho}$ is the contribution from fig. $3(\mathrm{~d})$ and $R_{\mu \sigma}^{a c \rho}$ represents the remaining terms that are regular when $y=-1$. Using standard Feynman rules one finds

$$
D_{\mu \sigma}^{a c \rho}=\left[-i f^{a c b} V_{\mu \sigma \alpha}\left(k_{1},-k_{2}, k_{2}-k_{1}\right)\right]\left[\frac{-\delta^{b d} P_{\beta}^{\alpha}\left(k_{1}-k_{2}\right)}{\left(k_{1}-k_{2}\right)^{2}}\right] B^{d \beta \rho}\left(q, k_{1}-k_{2}\right),
$$

with

$$
V_{\mu \sigma \alpha}\left(k_{1},-k_{2}, k_{2}-k_{1}\right)=\left(k_{1}+k_{2}\right)_{\alpha} g_{\mu \sigma}+\left(k_{1}-2 k_{2}\right)_{\mu} g_{\sigma \alpha}+\left(k_{2}-2 k_{1}\right)_{\sigma} g_{\mu \alpha},
$$

and $B^{b \alpha \beta}$ the sum of the lowest order diagrams in fig. 2. We choose the propagator in the light-like axial gauge where

$$
P_{\mu \nu}(k)=-g_{\mu \nu}+\frac{k_{\mu} \eta_{\nu}+k_{\nu} \eta_{\mu}}{\eta \cdot k} .
$$

Now $\eta$ and $k_{1}$ define a plane. Take $k_{\perp}$ perpendicular to this plane and choose $\eta$ such that $\eta \cdot k_{1} \neq 0$ and $\eta^{2}=0$. Then decomposing $k_{2}=(1-x) k_{1}+\eta \xi+k_{\perp}$ implies that $\xi=-k_{\perp}^{2} /\left[(1-x) 2 \eta \cdot k_{1}\right]$ and $t^{\prime}=k_{\perp}^{2} /(1-x)$. The collinear limit we require is $k_{\perp} \rightarrow 0$ with $x$ fixed. If we use $\epsilon\left(k_{1}\right) \cdot k_{1}=0, \bar{\epsilon}\left(k_{2}\right) \cdot k_{2}=0$, and $\left(k_{1}-k_{2}\right)_{\alpha} P_{\beta}^{\alpha}\left(k_{1}-k_{2}\right)=O\left(k_{\perp}^{2}\right)$ then

$$
V^{\mu \sigma \alpha}\left(k_{1},-k_{2}, k_{2}-k_{1}\right) \simeq \frac{2}{x} k_{\perp}^{\alpha} g^{\mu \sigma}-2 k_{\perp}^{\mu} g^{\sigma \alpha}+\frac{2}{1-x} k_{\perp}^{\sigma} g^{\mu \alpha}+\cdots+O\left(k_{\perp}^{2}\right),
$$

where $\cdots$ represents terms that vanish upon contraction with the polarization tensors. Hence (C.1) becomes

$$
\begin{aligned}
M^{a c \rho} & =\left\{-i g f^{a c b}\left[\frac{2}{x} k_{\perp \alpha} g_{\mu \sigma}-2 k_{\perp \mu} g_{\sigma \alpha}+\frac{2}{1-x} k_{\perp \sigma} g_{\mu \alpha}+\cdots+O\left(k_{\perp}^{2}\right)\right]\right. \\
& \left.\times\left[\frac{-\delta^{b d} P_{\beta}^{\alpha}\left(k_{1}-k_{2}\right)}{\left(k_{1}-k_{2}\right)^{2}}\right] B^{d \beta \rho}\left(q, k_{1}-k_{2}\right)+R_{\mu \sigma}^{a c \rho}\right\} \epsilon^{\mu}\left(k_{1}\right) \bar{\epsilon}^{\sigma}\left(k_{2}\right) .
\end{aligned}
$$

From this result one can see that $M \sim O\left(1 / \sqrt{t^{\prime}}\right)+R+O\left(t^{\prime}\right)$ as $t^{\prime} \rightarrow 0$. Hence when we square $M$ we can drop interference terms containing $R$ because only the square 
of the first term gives a $1 / t^{\prime}$ singularity. Now because $k_{1}-k_{2}=x k_{1}+O\left(k_{\perp}\right)$ we have $B^{a \beta \rho}\left(q, k_{1}-k_{2}\right)=B^{a \beta \rho}\left(q, x k_{1}\right)+O\left(k_{\perp}\right)$ so we can write

$$
\begin{aligned}
M^{a c \rho}\left(M^{a c \lambda}\right)^{\dagger} & =-\frac{4 g^{2} C_{A}}{t^{\prime}} B^{a \beta \rho}\left(q, x k_{1}\right)\left(B^{a \alpha \lambda}\left(q, x k_{1}\right)\right)^{\dagger} P_{\alpha \beta}\left(k_{1}\right) \\
& \times\left[(1-x)+\frac{1}{1-x}+\frac{1-x}{x^{2}}\right] \\
& +\frac{4 g^{2} C_{A}}{t^{\prime}} B^{a \beta \rho}\left(q, x k_{1}\right)\left(B^{a \alpha \lambda}\left(q, x k_{1}\right)\right)^{\dagger} \frac{(1-x)(n-2)}{x^{2}} \\
& \times\left[\frac{k_{\perp \alpha} k_{\perp \beta}}{k_{\perp}^{2}}+\frac{P_{\alpha \beta}}{n-2}\right] .
\end{aligned}
$$

Using the definition of $f_{i}^{g}\left(x, y, \theta_{1}, \theta_{2}\right)$ in eq. (3.3) one can easily make the identification used in the main text, namely that

$$
f_{i}^{g}\left(x,-1, \theta_{1}, \theta_{2}\right)=f_{i}^{g}\left(x, \theta_{1}\right)+\tilde{f}_{i}^{g}\left(x, \theta_{1}, \theta_{2}\right) .
$$

The second term vanishes upon integration over $\theta_{2}$ and

$$
\begin{aligned}
f_{i}^{g}\left(x, \theta_{1}\right) & =512 \pi^{2} \mu^{-2 \epsilon} \alpha_{s}^{2} e^{2} e_{H}^{2} N C_{A} C_{F} \frac{\left(s^{\prime}\right)^{2}}{s} \\
& \times \frac{1-x}{x}\left[x(1-x)+\frac{x}{1-x}+\frac{1-x}{x}\right] B_{i, Q E D}\left(x k_{1}\right) .
\end{aligned}
$$

The mass parameter $\mu$ originates from the dimensionality of the gauge coupling in $n$ dimensions. For the quark channel a similar analysis holds with the result

$$
f_{i}^{q}\left(x,-1, \theta_{1}, \theta_{2}\right)=f_{i}^{q}\left(x, \theta_{1}\right)+\tilde{f}_{i}^{q}\left(x, \theta_{1}, \theta_{2}\right)
$$

where again the second term vanishes upon integration over $\theta_{2}$ and

$$
\begin{aligned}
f_{i}^{q}\left(x, \theta_{1}\right) & =-128 \pi^{2} \mu^{-2 \epsilon} \alpha_{s}^{2} e^{2} e_{H}^{2} N C_{F}(1+\epsilon / 2)^{-1} \\
& \times\left[\frac{1+(1-x)^{2}+\epsilon x^{2} / 2}{x^{2}}\right] B_{i, Q E D}\left(x k_{1}\right) .
\end{aligned}
$$

Note that we need the full $f_{i}^{g}\left(x,-1, \theta_{1}, \theta_{2}\right)$ in $n=4$ dimensions in the plus distributions used in the main text not just $f_{i}^{g}\left(x, \theta_{1}\right)$. For this one may take the $n=4$ limit of eq. (C.7) by using an explicit representation of $k_{\perp}$. However, we found it instructive to take the collinear limit of $M_{i}^{g}(3)$ in the Feynman gauge and compare with the axial gauge results. It is well known [32] that if one chooses a gauge different from the axial one in QCD, then interference graphs also become singular in the collinear limit. The axial gauge result follows from the previous analysis. In the Feynman gauge the squared matrix element arising from the diagrams in fig. 3 (including interference terms) has the form

$$
M_{i}^{g}(3)=\frac{A}{\left(t^{\prime}\right)^{2}}+\frac{B}{t^{\prime}}+C+\mathcal{O}\left(t^{\prime}\right)
$$


where $A, B$, and $C$ are functions of the other nine Mandelstam invariants defined in appendix A. Expanding the invariants $u_{1}, u_{7}$, and $t_{1}$ about $t^{\prime}=0$ we find

$$
\begin{aligned}
u_{1} & =u_{1}^{c}-\frac{t^{\prime}}{s_{5}^{\prime}}\left[u_{1}^{c}+Q^{2} \beta_{5} \cos \theta_{1}\right], \\
u_{7} & =x t_{1}^{c}-\frac{t^{\prime}}{s_{5}^{\prime}}\left[x t_{1}^{c}-Q^{2} \beta_{5} \cos \theta_{1}\right], \\
t_{1} & =t_{1}^{c}-\frac{s^{\prime}}{2} \sqrt{2 a t^{\prime}} \beta_{5} \cos \theta_{2} \sin \theta_{1} \\
& +\frac{t^{\prime}}{s_{5}^{\prime}}\left\{u_{1}^{c}+\beta_{5} \cos \theta_{1}\left[Q^{2}\left(\frac{x-1}{x}\right)+s^{\prime}\right]\right\},
\end{aligned}
$$

where

$$
a=-2(1-x) \frac{s_{5}}{\left(x s^{\prime}\right)^{2}}
$$

and

$$
\begin{aligned}
t_{1}^{c} & =-\frac{1}{2} s^{\prime}\left(1-\beta_{5} \cos \theta_{1}\right), \\
u_{1}^{c} & =-\frac{1}{2} s_{5}^{\prime}\left(1+\beta_{5} \cos \theta_{1}\right) .
\end{aligned}
$$

Direct substitution of the invariants (C.13) into (C.12) and using the definition of $f_{G}^{g}\left(x, y, \theta_{1}, \theta_{2}\right)$ in eq. (3.3) yields the results

$$
\begin{aligned}
f_{G}^{g}\left(x,-1, \theta_{1}, \theta_{2}\right) & =-\frac{32\left(2 m^{2}-Q^{2}\right)\left(x s^{\prime}\right)^{2}}{\left(x t_{1}^{c}\right) u_{1}^{c} s} g\left(x, \theta_{1}, \theta_{2}\right) \\
& +\frac{32\left(x^{2}-x+1\right)^{2}\left(x s^{\prime}\right)^{2}}{x^{4} s}\left[\frac{\left(x s^{\prime}\right)^{2}}{\left(x t_{1}^{c}\right) u_{1}^{c}}-2\right] \\
f_{L}^{g}\left(x,-1, \theta_{1}, \theta_{2}\right) & =-\frac{64 Q^{2}}{s} g\left(x, \theta_{1}, \theta_{2}\right)
\end{aligned}
$$

where

$$
\begin{aligned}
g\left(x, \theta_{1}, \theta_{2}\right) & =\frac{2\left(x^{2}-2 x+2\right)}{x^{2}}\left[m^{2} \frac{\left(x s^{\prime}\right)^{2}}{\left(x t_{1}^{c}\right) u_{1}^{c}}-s_{5}\right] \\
& -\frac{(1-x)^{2}}{x^{4}} s_{5} \beta_{5}^{2} \cos ^{2} \theta_{2} \sin ^{2} \theta_{1} \frac{\left(x s^{\prime}\right)^{2}}{\left(x t_{1}^{c}\right) u_{1}^{c}} .
\end{aligned}
$$

Both $f_{G}^{g}\left(x,-1, \theta_{1}, \theta_{2}\right)$ and $f_{L}^{g}\left(x,-1, \theta_{1}, \theta_{2}\right)$ have a common factor $g^{4} e^{2} e_{H}^{2} N C_{A} C_{F}$ which we have not shown. We have used the relation $x s^{\prime}+x t_{1}^{c}+u_{1}^{c}=0$ freely in deriving (C.16) and (C.17). A similar analysis for the quark channel gives the 
results

$$
\begin{aligned}
& f_{G}^{q}\left(x,-1, \theta_{1}, \theta_{2}\right)=\frac{16\left(2 m^{2}-Q^{2}\right)}{\left(x t_{1}^{c}\right) u_{1}^{c}} h\left(x, \theta_{1}, \theta_{2}\right) \\
&-\frac{8\left(x^{2}-2 x+2\right)}{x^{2}}\left[\frac{\left(x s^{\prime}\right)^{2}}{\left(x t_{1}^{c}\right) u_{1}^{c}}-2\right] \\
& f_{L}^{q}\left(x,-1, \theta_{1}, \theta_{2}\right)=\frac{32 Q^{2}}{\left(x s^{\prime}\right)^{2}} h\left(x, \theta_{1}, \theta_{2}\right)
\end{aligned}
$$

where

$$
h\left(x, \theta_{1}, \theta_{2}\right)=m^{2} \frac{\left(x s^{\prime}\right)^{2}}{\left(x t_{1}^{c}\right) u_{1}^{c}}-s_{5}-\frac{(1-x)}{x^{2}} s_{5} \beta_{5}^{2} \cos ^{2} \theta_{2} \sin ^{2} \theta_{1} \frac{\left(x s^{\prime}\right)^{2}}{\left(x t_{1}^{c}\right) u_{1}^{c}} .
$$

Both $f_{G}^{q}\left(x,-1, \theta_{1}, \theta_{2}\right)$ and $f_{L}^{q}\left(x,-1, \theta_{1}, \theta_{2}\right)$ have a common factor $g^{4} e^{2} e_{H}^{2} N C_{F}$ which we have not shown. We have checked the formulae for the $f_{i}^{a_{1}}\left(x,-1, \theta_{1}, \theta_{2}\right)\left(a_{1}=\right.$ $g, q, i=G, L)$ presented above by comparing them numerically with the $y \rightarrow-1$ limit of the complete expression $f_{i}^{a_{1}}\left(x, y, \theta_{1}, \theta_{2}\right)$. 


\section{Figure Captions}

fig.1. Illustration of the basic reaction (2.1) for heavy-flavor production in virtualphoton-parton collisions.

fig.2. Lowest order Feynman diagram contributing to the amplitude for the gluon fusion reaction (2.12). Another diagram is obtained by reversing the arrows on the heavy quark lines.

fig.3. The order $e g^{2}$ diagrams contributing to the amplitude for the gluon-bremsstrahlung reaction (3.1). Additional graphs are obtained by reversing the arrows on the heavy quark lines.

fig.4. One loop order $e g^{3}$ diagrams for the virtual corrections contributing to the reaction (2.12). Except for the non-planar diagram additional diagrams are obtained by reversing the arrows on the heavy quark lines. The solid line in the gluon self-energy graph represents gluon, ghost and quark loops.

fig.5. The order $e g^{2}$ diagrams contributing to the amplitude for the reaction (3.30). Additional graphs are obtained by reversing the arrows on the light-quark lines (dashed).

fig.6. The $\eta$ dependence of the scaling function $c_{L, q}^{(1)}(\eta, \xi)$ in the $\overline{\mathrm{MS}}$ scheme for several values of $Q^{2}$ (in units of $(\mathrm{GeV} / \mathrm{c})^{2}$ ) with $m=4.75\left(\mathrm{GeV} / c^{2}\right)$, i. e. the corrected version of fig. 9(b) of ref. [1]. The solid line corresponds to $Q^{2}=0.01$, the dotted line to $Q^{2}=1$, the short dashed line to $Q^{2}=10$, the long dashed line to $Q^{2}=100$, and the dot-dashed line to $Q^{2}=1000$.

fig.7. (a) The $\eta$ dependence of the scaling function $d_{T, q}^{(1)}(\eta, \xi)$ in the $\overline{\mathrm{MS}}$ scheme for several values of $Q^{2}$ (in units of $\left.(\mathrm{GeV} / \mathrm{c})^{2}\right)$ with $m=4.75\left(\mathrm{GeV} / c^{2}\right)$, i.e. the corrected version of fig. 11(a) of ref. [1]. The short dashed line corresponds to $Q^{2}=10$, the long dashed line to $Q^{2}=100$, and the dot-dashed line to $Q^{2}=1000$. (b) The $\eta$ dependence of the scaling function $d_{L, q}^{(1)}(\eta, \xi)$ in the $\overline{\mathrm{MS}}$ scheme for the same values of $Q^{2}$ with $m=4.75\left(\mathrm{GeV} / c^{2}\right)$ i.e. the corrected version of fig. 11(b) of ref. [1].

fig.8. (a) The distributions $d F_{2}\left(x, Q^{2}, m_{c}^{2}, P_{t}\right) / d P_{t}$ for charm-anticharm pair production at fixed $Q^{2}=12(\mathrm{GeV} / \mathrm{c})^{2}$ with $x=4.2 \times 10^{-4}$ (solid line), $8.5 \times 10^{-4}$ (dotted line), $1.6 \times 10^{-3}$ (short dashed line) and $2.7 \times 10^{-3}$ (long dashed line). (b) The same distributions at fixed $x=8.5 \times 10^{-4}$ and $Q^{2}=8.5$ (solid line), 12 (dotted line), 25 (short dashed line), 50 (long dashed line) all in units of $(\mathrm{GeV} / \mathrm{c})^{2}$.

fig.9. (a) The distributions $d F_{2}\left(x, Q^{2}, m_{c}^{2}, \Delta \phi\right) / d(\Delta \phi)$ for charm-anticharm pair production at the $x$ and $Q^{2}$ values given in fig. $8(\mathrm{a})$. (b) The same distributions at the $x$ and $Q^{2}$ values given in fig. 8(b). 
fig.10. (a) The distributions $d F_{2}\left(x, Q^{2}, m_{c}^{2}, R\right) / d R$ for charm-anticharm pair production at the $x$ and $Q^{2}$ values given in fig. 8(a). (b) The same distributions at the $x$ and $Q^{2}$ values given in fig. 8(b).

fig.11. (a) The distributions $d F_{L}\left(x, Q^{2}, m_{c}^{2}, P_{t}\right) / d P_{t}$ for charm-anticharm pair production at the $x$ and $Q^{2}$ values given in fig. 8(a). (b) The same distributions at the $x$ and $Q^{2}$ values given in fig. 8(b).

fig.12. (a) The distributions $d F_{L}\left(x, Q^{2}, m_{c}^{2}, \Delta \phi\right) / d(\Delta \phi)$ for charm-anticharm pair production at the $x$ and $Q^{2}$ values given in fig. 8(a). (b) The same distributions at the $x$ and $Q^{2}$ values given in fig. 8(b).

fig.13. (a) The distributions $d F_{L}\left(x, Q^{2}, m_{c}^{2}, R\right) / d R$ for charm-anticharm pair production at the $x$ and $Q^{2}$ values given in fig. 8(a). (b) The same distributions at the $x$ and $Q^{2}$ values given in fig. 8(b).

fig.14. (a) The distributions $d F_{2}\left(x, Q^{2}, m_{b}^{2}, P_{t}\right) / d P_{t}$ for bottom-antibottom pair production at the $x$ and $Q^{2}$ values given in fig. 8(a). (b) The same distributions at the $x$ and $Q^{2}$ values given in fig. 8(b).

fig.15. (a) The distributions $d F_{2}\left(x, Q^{2}, m_{b}^{2}, \Delta \phi\right) / d(\Delta \phi)$ for bottom-antibottom pair production at the $x$ and $Q^{2}$ values given in fig. 8(a). (b) The same distributions at the $x$ and $Q^{2}$ values given in fig. 8(b).

fig.16. (a) The distributions $d F_{2}\left(x, Q^{2}, m_{b}^{2}, R\right) / d R$ for bottom-antibottom pair production at the $x$ and $Q^{2}$ values given in fig. 8(a). (b) The same distributions at the $x$ and $Q^{2}$ values given in fig. 8(b).

fig.17. (a) The distributions $d F_{L}\left(x, Q^{2}, m_{b}^{2}, P_{t}\right) / d P_{t}$ for bottom-antibottom pair production at the $x$ and $Q^{2}$ values given in fig. $8(\mathrm{a})$. (b) The same distributions at the $x$ and $Q^{2}$ values given in fig. $8(\mathrm{~b})$.

fig.18. (a) The distributions $d F_{L}\left(x, Q^{2}, m_{b}^{2}, \Delta \phi\right) / d(\Delta \phi)$ for bottom-antibottom pair production at the $x$ and $Q^{2}$ values given in fig. 8(a). (b) The same distributions at the $x$ and $Q^{2}$ values given in fig. 8(b).

fig.19. (a) The distributions $d F_{L}\left(x, Q^{2}, m_{b}^{2}, R\right) / d R$ for bottom-antibottom pair production at the $x$ and $Q^{2}$ values given in fig. 8(a). (b) The same distributions at the $x$ and $Q^{2}$ values given in fig. 8(b). 


\section{References}

[1] E. Laenen, S. Riemersma, J. Smith and W. L. van Neerven, Nucl. Phys. B392 (1993) 162.

[2] E. Laenen, S. Riemersma, J. Smith and W. L. van Neerven, Nucl. Phys. B392 (1993) 229.

[3] E. B. Zijlstra and W. L. van Neerven, Nucl. Phys. B383, 525 (1992).

[4] E. Laenen, S. Riemersma, J. Smith and W. L. van Neerven, Phys. Lett. B291 (1992) 325.

[5] S. Riemersma, J. Smith and W. L. van Neerven, Phys. Lett. B347 (1995) 143.

[6] B. W. Harris and J. Smith, ITP-SB-94-06, to appear in Phys. Lett. B.

[7] H. L. Lai, J. Botts, J. Huston, J. G. Morfín, J. F. Owens, J. W. Qiu, W-K. Tung and H. Weerts, MSU-HEP-41024 (Oct. 1994).

[8] H1 Collaboration, I. Abt et al., Nucl. Phys. B407 (1993) 515; ZEUS Collaboration, M. Derrick et al., Z. Phys C65 (1995) 379.

[9] R. K. Ellis and P. Nason, Nucl. Phys. B312 (1989) 551; J. Smith and W. L. van Neerven, Nucl. Phys. B374 (1992) 36; W. L. van Neerven, Nucl. Phys. B29A (Proc. Suppl.) (1992) 199; S. Frixione, M. L. Mangano, P. Nason and G. Ridolfi, CERN-TH.7527/94.

[10] S. Frixione, M. L. Mangano, P. Nason and G. Ridolfi, Nucl. Phys. B412 (1994) 225 .

[11] ZEUS Collaboration, M. Derrick et al., Study of $D^{*}(2010)^{+}$production in $e P$ collisions at HERA, DESY 95-013.

[12] G. A. Schuler, Nucl. Phys. B299 (1988) 21; A. Ali, G. Ingelman, G.A. Schuler, F. Barreiro, M.A. García, J.F. de Trocóniz, R.A. Eichler and Z. Kunszt, Heavyquark physics at HERA, Proc. DESY workshop on HERA physics, DESY, Hamburg(1988), DESY 88-119; A. Ali and D. Wyler in Physics at HERA, vol 2 p. 669, Proceedings of the Workshop Hamberg, Oct 29 (1991) Eds. W. Buchmüller and G. Ingelman; A. Ali, DESY 93-105.

[13] S. J. Brodsky, P. Hoyer, A. H. Mueller and W-K. Tang, Nucl. Phys. B369 (1992) 519; G. Ingelman, L. Jönsson and M. Nyborg, Phys. Rev. D47 (1993) 4872. For a complete list of references see R. Vogt and S. J. Brodsky LBL-35380 (1994).

[14] M. A. G. Aivazis, F. I. Olness and W-K. Tung, Phys. Rev. D50 (1994) 3085; M. A. G. Aivazis, J. C. Collins, F. I. Olness and W-K. Tung, Phys. Rev. D50 3102 (1994). 
[15] F. I. Olness and S. Riemersma, SMU-HEP/94-21, to appear in Phys. Rev. D.

[16] R. K. Ellis, D. A. Ross and A. E. Terrano, Nucl. Phys. B178 (1981) 421; Z. Kunszt and P. Nason, in Z Physics LEP 1, Proceedings of the Workshop, Geneva, Switzerland, 1989, ed. by G. Altarelli, R. Kleiss and C. Verzegnassi (CERN, Geneva, 1989), Vol. 1.

[17] B. Mele, P. Nason and G. Ridolfi, Nucl. Phys. 357 (1991) 409.

[18] Z. Kunszt and D. E. Soper, Phys. Rev. D46 (1992) 192.

[19] M. Mangano, P. Nason and G. Ridolfi, Nucl. Phys. B373 (1992) 295.

[20] S. Mendoza and J. Smith, Phys. Rev. D50 (1994) 226; Phys. Rev. D50 (1994) 251.

[21] H. Baer, J. Ohnemus and J. F. Owens, Phys. Rev. D40 (1989) 2844.

[22] J. C. Collins, D. E. Soper and G. Sterman, Nucl. Phys. B263 (1986) 37. For a review see J. C. Collins, D. E. Soper and G. Sterman in Perturbative QCD ed. A. H. Mueller ( World Scientific, 1989).

[23] G. P. Lepage, J. Comp. Phys. 27 (1978) 192.

[24] J. A. M. Vermaseren, FORM Version 2.2b, CAN, Amsterdam, The Netherlands, 1991.

[25] G. Altarelli and G. Parisi, Nucl. Phys. B126 (1977) 298; V. N. Gribov and L. N. Lipatov, Yad. Fiz. 15 (1972) 1218 [Sov. J. Nucl. Phys. 15 (1972) 675].

[26] J. C. Collins, F. Wilczek and A. Zee, Phys. Rev. D18 (1978) 242; J. C. Collins and W-K. Tung, Nucl. Phys. B278 (1986) 934.

[27] J. Kubar André and F. E. Paige, Phys. Rev. D19 (1978) 221; B. Humpert and W. L. van Neerven, Nucl. Phys. B184 (1981) 225.

[28] T. Muta, Foundations of Quantum Chromodynamics: An Introduction to Perturbative Methods in Gauge Theories (World Scientific, New Jersey, 1987), p. 346 .

[29] W. Beenakker, H. Kuijf, W. L. van Neerven and J. Smith, Phys. Rev. D40 (1989) 54.

[30] W. L. van Neerven, Nucl. Phys. B268 (1986) 453.

[31] L. Lewin, Polylogarithms and associated functions, ( North-Holland, Amsterdam, 1983 ).

[32] B. Humpert and W. L. van Neerven, Nucl. Phys. B178 (1981) 498. 